\title{
A THEORY OF SOME MULTIPLE DECISION PROBLEMS*. II
}

\author{
By E. L. Lehmann ${ }^{1}$ \\ University of California, Berkeley
}

Summary. The theory of Part $I$ is extended to problems in which it is permitted not to come to a definite conclusion regarding one or more of the questions under consideration. Some problems are also investigated in which, from a single set of observations, one wishes to answer a number of questions in sequence. Here the nature of the question at a later stage will depend on the answers obtained at the earlier stages.

9. Decision procedures permitting partial conclusions. It is a common feature of all the problems treated in Part I, that a fixed partition of the parameter space $\Omega$ into sets $\Omega_{t}$ is given, and that ore wishes to determine which of these sets contains the true parameter point. There are however many statistical problems, such as the estimation by confidence sets, in which the possible decisions do not correspond to the sets of a fixed partition. In particular, this is the case in the field of statistical inference, when the statistician is free to decide how sharp a statement he can reliably make on the basis of the observations. We shall show in the present section how such problems may be generated by the simultaneous consideration of a number of two-decision problems as in Part I, if one suitably modifies the interpretation of the decisions involved.

Previously we were concerned with testing a set of hypotheses $H_{\gamma}: \theta \varepsilon \omega_{\gamma}$, so that in each component problem the choice lay between the two decisions $\theta \varepsilon \omega_{\gamma}$ (acceptance of $H_{\gamma}$ ) and $\theta \varepsilon \omega_{\gamma}^{-1}$ (rejection of $H_{\gamma}$ ). Suppose now instead that the statistician is asked only whether the data reject the hypothesis, and that in case they do not, no alternative positive statement is required. The choice may then be said to lie between the statements $\theta \varepsilon \omega_{\gamma}^{-1}$ and $\theta \varepsilon \omega_{\gamma}^{0}=\Omega$.

This actually appears to be the point of view taken by Duncan ("Multiple range and multiple F tests", Biometrics, Vol. 11 (1955), pp. 1-42) in his formulation of this class of multiple decision problems to which reference is made in section 1 of the present paper.

If one considers simultaneously a number of such problems, one is faced with a multiple decision problem in which the different possible decisions correspond to the statements that a certain number of the hypotheses $H_{\gamma}$ are false, but where nothing is said regarding the remaining hypotheses. This is equivalent to the statement that the parameter point $\theta$ lies in the set

$$
\Omega_{i}=\bigcap_{\gamma} \omega_{\gamma}^{y_{i}}
$$

Received October 12, 1956.

* To rectify a printing error in Part $I$, in the March, 1957 issue of these Annals replace page 14, formula (4.12) through page 17, line 23 by page 70, line 8 through page 72, nextto-last line. Ed.

${ }^{1}$ Work done while the author was a Fellow of the John Simon Guggenheim Memorial Foundation. 
where the $y$ 's are -1 for each rejected $H_{\gamma}$ and 0 for the others. In the particular case that all of the $y$ 's are zero, we have $\Omega_{i}=\Omega$, and thus make no statement whatever about the position of $\theta$. As before, it may of course happen that some of the formal intersections (9.1) are empty, and we shall then restrict the $\Omega$ 's to denote the nonempty ones and require that none of the possible decisions should correspond to these empty intersections. If we assume that in the simultaneous consideration of a number of problems the losses are additive so that the total loss is the sum (or a weighted sum) of the losses of the component problems, and if we suppose the losses to be $a_{\gamma}$ for rejecting $H_{\gamma}$ when $\theta \varepsilon \omega_{\gamma}, b_{\gamma}$ for not rejecting it when $\theta \varepsilon \omega_{\gamma}^{-1}$, and zero in the other two cases, the total loss is again given by $(2.2)$ and $(2.3)$ with

$$
x_{i \gamma}=2 y_{i \gamma}+1 \text {. }
$$

Since in particular the loss function of the basic two-decision problem is unchanged, the associated optimum unbiased procedure of Part I will retain its optimum property in spite of the reinterpretation of one of the decisions. It now leads to the statements $\theta \varepsilon \omega_{\gamma}^{-1}$ and $\theta \varepsilon \omega_{\gamma}^{0}$ as $X \varepsilon A_{\gamma}^{-1}$ or $X \varepsilon A_{\gamma}$ where $A_{\gamma}$ is the acceptance region of the best unbiased test of $H_{\gamma}$. The simultaneous carrying out of a number of these tests then still leads to a procedure in which the decision $d_{i}: \theta \varepsilon \Omega_{i}$ is taken when $X$ falls in the set

$$
D_{i}=\cap_{\gamma} A^{x_{i}}
$$

but where $\Omega_{i}$ is now defined by (9.1) and (9.2) instead of (2.1).

As has already been pointed out the sets $\Omega_{i}$, which define the possible decisions, no longer constitute a partition of the parameter space. Instead, they are generated through intersections from the class $\left\{\omega_{\gamma}^{-1}, \gamma \varepsilon \Gamma\right\}$, that is, they constitute the smallest class that is closed under intersections and contains the sets $\omega_{\gamma}^{-1}$. It may happen that two of these $\Omega^{\prime}$ 's are equal, $\Omega_{i}=\Omega_{j}$, say, and one would then wish to identify the associated decisions. On the other hand, viewing the problem as a product one must consider all of the formal intersections (9.1) as distinct. Otherwise the definition of the lass function, for example, would become ambiguous since the losses resulting from decisions $d_{i}$ and $d_{j}$ when $\theta$ is in some $\Omega_{h}$ would usually not be the same even though $\Omega_{i}=\Omega_{j}$. Fortunately, the difficulty arises in the applications we wish to make only in cases in which it can be overcome by a natural further restriction of the decision space. Suppose namely that $H_{\gamma}$ and $H_{\delta}$ are two hypotheses with

$$
\omega_{\gamma}^{-1} \subset \omega_{\delta}^{-1}
$$

so that the two intersections $\omega_{\gamma}^{-1} \cap \omega_{\delta}^{-1}$ and $\omega_{\gamma}^{-1} \cap \omega_{\delta}^{0}$ are identical. It then seems reasonable that whenever the data lead to the rejection of $H_{\gamma}$ one would also wish to reject the more restrictive hypothesis $H_{\delta}$. (In part I this was actually part of the compatibility requirement.) With such tests the decisions $\theta \varepsilon \omega_{\gamma}^{-1} \cap \omega_{\delta}^{0}$ would never be reached, and the conflict would thus be avoided. For this reason we shall eliminate from the list of permissible decisions not only the formal intersections 
(9.1) that are empty, but also those for which the intersection $\Omega_{i}$ equals some other $\Omega_{j}$ satisfying $y_{j \gamma} \leqq y_{i \gamma}$ for all $\gamma$. It follows from the general discussion of Sec. 7 that (9.3) defines a 1:1 correspondence between families of tests which are compatible with this set of restrictions, and decision procedures for the restricted product problem. It is useful to note further that for these restrictions the formation of restricted products of decision problems also retains the property of being commutative and associative, which it obviously had in the problems of Part I.

Let $P_{+}\left(\theta_{0}\right)$ denote the two-decision problem discussed above, in which the choice lies between the statements $\theta>\theta_{0}$ and $-\infty<\theta<\infty$, and let $P_{-}\left(\theta_{0}\right)$ denote the dual problem in which the first of these possibilities is replaced by the statement $\theta<\theta_{0}$. By considering these two problems simultaneously one obtains a three-decision problem with loss function

\begin{tabular}{|c|c|c|c|}
\hline & $\theta \stackrel{d_{2}}{<} \theta_{0}$ & $\begin{array}{c}d_{0} \\
-\infty<\theta<\infty\end{array}$ & $\begin{array}{c}d_{1} \\
\theta>\theta_{0}\end{array}$ \\
\hline$\theta<\theta_{0}$ & 0 & $b$ & $a+b$ \\
\hline$\theta=\theta_{0}$ & $a$ & 0 & $a$ \\
\hline$\theta>\theta_{0}$ & $a+b$ & $b$ & 0 \\
\hline
\end{tabular}

As an example suppose that $\theta$ measures the difference in quality of two products which are being compared by an impartial research organization. The decisions $d_{1}$ and $d_{2}$ claim superiority for one or the other of the products, while $d_{0}$ states that the data are inconclusive and that neither of the two products can be ascertained to be better than the other. It is an advantage of such a formulation over the more conventional one in which $d_{0}$ is replaced by the statement $\theta=\theta_{0}$, that it enables the statistician to control the probability of error. In the standard situation with $D_{2}$ and $D_{1}$ given by

$$
D_{2}: T \leqq C_{1} \text { and } D_{1}: T \geqq C_{1}^{\prime}
$$

where $P_{\theta}\left\{T \leqq C_{1}\right\}$ and $P_{\theta}\left\{T \geqq C_{2}^{\prime}\right\}$ are $<\alpha$ for $\theta>\theta_{0}$ and $\theta<\theta_{0}$ respectively, and where $P_{\theta_{0}}\left\{T \leqq C_{1}\right\}=P_{\theta_{0}}\left\{T \geqq C_{2}^{\prime}\right\}=\alpha$, the maximum probability of error occurs when $\theta=\theta_{0}$, and is $2 \alpha$. (A very similar formulation was discussed by Bahadur, "A property of the $t$-statistic," Sankhya, Vol. 12 (1952), pp. 79-88.)

The loss function (9.4) is not appropriate in situations in which a definite decision is preferred to $d_{0}$ even when $\theta=\theta_{0}$. A formulation which is more suitable for this case is obtained if in the one-sided problems $\theta_{-}\left(\theta_{0}\right)$ and $\theta_{+}\left(\theta_{0}\right)$ one replaces the decisions $\theta<\theta_{0}$ and $\theta>\theta_{0}$ by $\theta \leqq \theta_{0}$ and $\theta \geqq \theta_{0}$ respectively, so that these two component problems are given by

\begin{tabular}{c|cc} 
& $-\infty<\theta<\infty$ & $\theta \leqq \theta_{0}$ \\
\hline$\theta>\theta_{0}$ & 0 & $a$ \\
$\theta \leqq \theta_{0}$ & $b$ & 0
\end{tabular}


and

\begin{tabular}{c|cc} 
& $-\infty<\theta<\infty$ & $\theta \geqq \theta_{0}$ \\
\hline$\theta<\theta_{0}$ & 0 & $a$ \\
$\theta \geqq \theta_{0}$ & $b$ & 0
\end{tabular}

The simultaneous consideration of these two problems leads to a four-decision problem with loss table

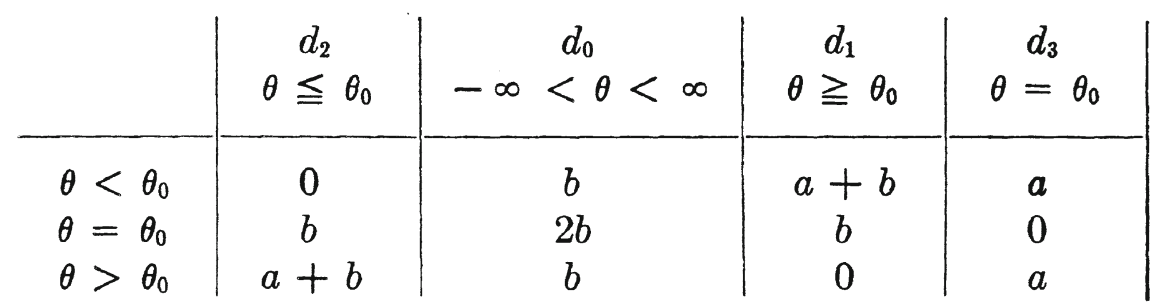

It turns out that this formulation leads to essentially the same solution as the previous one, with $D_{2}$ and $D_{1}$ given by (9.5), decision $d_{0}$ being taken when $C_{1}<T<C_{1}^{\prime}$, and decision $d_{3}$ not occurring at all.

We mention finally that still another problem leads to the same solution, namely that given by

$$
\left.\begin{array}{c|c|c|c|} 
& \begin{array}{c}
d_{2} \\
\theta \leqq \theta_{0}
\end{array} & -\infty<\theta<\infty & \begin{array}{c}
d_{0} \\
\theta \geqq \theta_{0}
\end{array} \\
\hline \theta<\theta_{0} & 0 & b & a^{\prime} \\
\theta=\theta_{0} & 0 & b^{\prime} & 0 \\
\theta>\theta_{0} & a^{\prime} & b & 0
\end{array} \quad \begin{array}{l}
b<a^{\prime} \\
b<b^{\prime}
\end{array}\right) .
$$

The level $\alpha$ of (9.5) is in this case given by

$$
\alpha=\frac{b^{\prime}-b}{a^{\prime}+2\left(b^{\prime}-b\right)} .
$$

10. Partial classification of one or more parameters. (i) Let $\theta$ be a real parameter and suppose that we wish to determine, as far as possible, its position relative to two given values $\theta_{1}<\theta_{2}$. A procedure may be generated by considering simultaneously the four problems $P_{ \pm}\left(\theta_{i}\right), i=1,2$. The resulting problem offers the choice between the decisions

$$
\begin{aligned}
& d_{1}:\left\{\theta<\theta_{1}\right\}=\left\{\theta<\theta_{1}\right\} \cap\left\{\theta<\theta_{2}\right\} \\
& d_{2}:\left\{\theta<\theta_{2}\right\}=\{-\infty<\theta<\infty\} \cap\left\{\theta<\theta_{2}\right\} \\
& d_{3}:\left\{\theta_{1}<\theta<\theta_{2}\right\}=\left\{\theta_{1}<\theta\right\} \cap\left\{\theta<\theta_{2}\right\} \\
& d_{4}:\left\{\theta>\theta_{1}\right\}=\left\{\theta>\theta_{1}\right\} \cap\{-\infty<\theta<\infty\} \\
& d_{5}:\left\{\theta>\theta_{2}\right\}=\left\{\theta>\theta_{2}\right\} \cap\left\{\theta>\theta_{1}\right\} \\
& d_{6}:\{-\infty<\theta<\infty\}=\{-\infty<\theta<\infty\} \cap\{-\infty<-\theta<\infty\} .
\end{aligned}
$$


Here the sets $\left\{\theta<\theta_{1}\right\}$ and $\left\{\theta>\theta_{2}\right\}$ may also be represented as the intersections $\left\{\theta<\theta_{1}\right\} \cap\{-\infty<\theta<\infty\}$ and $\left\{\theta>\theta_{2}\right\} \cap\{-\infty<\theta<\infty\}$. However these decisions are ruled out by the convention of the previous section.

Suppose now that the tests of the four generating hypotheses have rejection regions

$$
T \leqq C_{1}, \quad T \leqq C_{1}^{\prime}, \quad T \geqq C_{2}, \quad T \geqq C_{2}^{\prime}
$$

for $H: \theta \leqq \theta_{1}, \theta \geqq \theta_{1}, \theta \leqq \theta_{2}, \theta \geqq \theta_{2}$ respectively, where the constants are determined by

$$
P_{\theta_{1}}\left\{T \leqq C_{1}\right\}=P_{\theta_{1}}\left\{T \geqq C_{1}^{\prime}\right\}=P_{\theta_{2}}\left\{T \leqq C_{2}\right\}=P_{\theta_{2}}\left\{T \geqq C_{2}^{\prime}\right\}=\alpha .
$$

Compatibility requires that the intersections

$$
\begin{gathered}
\left\{T \leqq C_{i}\right\} \cap\left\{T \geqq C_{i}^{\prime}\right\}, \quad\left\{T \leqq C_{1}\right\} \cap\left\{C_{2}<T<C_{2}^{\prime}\right\} \\
\text { and }\left\{C_{1}<T<C_{i}^{\prime}\right\} \cap\left\{T \geqq C_{2}^{\prime}\right\}
\end{gathered}
$$

should be empty, and hence that

$$
C_{1}<C_{1}^{\prime}, \quad C_{2}<C_{2}^{\prime}, \quad C_{1}<C_{2} \text { and } C_{1}^{\prime}<C_{2}^{\prime} .
$$

These conditions are satisfied if $\alpha<\frac{1}{2}$, and if for each fixed $C$

$$
P_{\theta}\{T \leqq C\}>P_{\theta^{\prime}}\{T \leqq C\} \text { when } \theta<\theta^{\prime} .
$$

According to (9.3) the resulting procedure is given by

$$
\begin{array}{ll}
D_{1}: T \leqq C_{1} & D_{4}: \max \left(C_{1}^{\prime}, C_{2}\right) \leq T<C_{2}^{\prime} \\
D_{2}: C_{1} \leqq T<\min \left(C_{1}^{\prime}, C_{2}\right) & D_{5}: T \geqq C_{2}^{\prime} \\
D_{3}: C_{1}^{\prime} \leqq T \leqq C_{2} & D_{6}: C_{2}<T<C_{1}^{\prime}
\end{array}
$$

where $\leq$ is $<$ when $C_{1}^{\prime}<C_{2}$ and $\leqq$ when $C_{1}^{\prime} \geqq C_{2}$. Depending on the sign of the difference $C_{2}-C_{1}^{\prime}$ and hence on the distance between $\theta_{1}$ and $\theta_{2}$, only one of the decisions $d_{3}$ and $d_{6}$ will occur. For intermediate values of $T$ the positive statement $\theta_{1}<\theta<\theta_{2}$ will be made only if $\theta_{1}$ and $\theta_{2}$ are not too close. Otherwise such $T$-values will leave the position of $\theta$ in doubt.

If (10.3) holds, the probability of the procedure leading to a false statement never exceeds $2 \alpha$. For the probability of error is equal to

$$
\begin{array}{lr}
P_{\theta}\left\{T \geqq C_{1}^{\prime}\right\} \leqq P_{\theta_{1}}\left\{T \geqq C_{1}^{\prime}\right\}=\alpha & \text { for } \theta<\theta_{1} \\
P_{\theta_{1}}\left\{T \leqq C_{1}\right\}+P_{\theta_{1}}\left\{T \geqq C_{1}^{\prime}\right\}=2 \alpha & \text { for } \theta=\theta_{1} \\
P_{\theta}\left\{T \leqq C_{1}\right\}+P_{\theta}\left\{T \geqq C_{2}\right\} \leqq P_{\theta_{1}}\left\{T \leqq C_{1}\right\}+P_{\theta_{2}}\left\{T \geqq C_{2}\right\}=2 \alpha \\
& \text { for } \quad \theta_{1}<\theta<\theta_{2},
\end{array}
$$

and similarly for $\theta \geqq \theta_{2}$.

In the usual applications $T$ is a function of a sample, and as the sample size increases $T$ tends to $\theta$ in probability. The procedure is then consistent in the 
sense that

$$
P\left(D_{i}\right) \rightarrow 1 \text { for } \theta \varepsilon \Omega_{i} \quad(i=1,2,3) .
$$

Except when $\theta$ is exactly equal to $\theta_{1}$ or $\theta_{2}$, the probability is therefore 1 that one of the three sharp statements $d_{1}, d_{3}$, or $d_{5}$ will be made, and that this statement will be correct. Since

$$
P_{\theta_{1}}\left(D_{2}\right)=P_{\theta_{2}}\left(D_{4}\right)=1-2 \alpha
$$

it also follows that

$$
P_{\theta_{1}}\left(D_{2}\right)=P_{\theta_{2}}\left(D_{4}\right) \rightarrow 1
$$

if one lets $\alpha$ tend to zero as $n$ tends to infinity.

A slightly different procedure results for problem (10.1) when one is concerned with a sample $X_{1}, \cdots, X_{n}$ from $N\left(\theta, \sigma^{2}\right)$. Here the tests of the four generating hypotheses have rejection regions

$$
\begin{aligned}
\left(\bar{X}-\theta_{1}\right) / S \leqq-C, \quad\left(\bar{X}-\theta_{1}\right) / S \geqq C, \\
\left(\bar{X}-\theta_{2}\right) / S \leqq-C, \quad\left(\bar{X}-\theta_{2}\right) / S \geqq C,
\end{aligned}
$$

and the induced multiple decision procedure is given by $(10.3)$ with

$$
T=\bar{X} / S, \quad C_{i}=-C+\frac{\theta_{i}}{S}, \quad C_{i}^{\prime}=C+\frac{\theta_{i}}{S}
$$

Both of the decisions $d_{3}$ and $d_{6}$ occur in this procedure with positive probability, $d_{3}$ only in cases in which $S \leqq\left(\theta_{2}-\theta_{1}\right) / C$ and $d_{6}$ only when the opposite inequality holds. The remarks concerning error control and consistency require no change.

As an application consider the comparison of two normal populations $N\left(\xi, \sigma^{2}\right)$ and $N\left(\eta, \sigma^{2}\right)$ on the basis of samples $X_{1}, \cdots, X_{m}$ and $Y_{1}, \cdots, Y_{n}$. With $\theta=\eta-\xi, \theta_{1}=-\Delta, \theta_{2}=\Delta$, the possible decisions become

$$
\begin{array}{lll}
d_{1}: \eta-\xi<-\Delta, & d_{3}:-\Delta<\eta-\xi<\Delta, & d_{5}: \eta-\xi>\Delta, \\
d_{2}: \eta-\xi<\Delta, & d_{4}: \eta-\xi>-\Delta, & d_{6}:-\infty<\eta-\xi<\infty .
\end{array}
$$

Here $d_{1}$ states that $\xi$ is significantly larger than $\eta, d_{2}$ that $\eta$ is not significantly larger than $\xi, d_{3}$ that the two means do not differ significantly, etc. The procedure is given by $(10.3)$ with $T=(\bar{Y}-\bar{X}) / S$ and

$$
C_{1}=-C-\frac{\Delta}{\bar{S}}, \quad C_{2}=-C+\frac{\Delta}{S}, \quad C_{1}^{\prime}=C-\frac{\Delta}{\bar{S}}, \quad C_{2}^{\prime}=C+\frac{\Delta}{S} .
$$

Problem (10.1) leads to still another type of procedure in the case of two independent Poisson variables, say $X$ and $Y$, where one wishes to classify the ratio $\rho=\lambda / \mu$ of the parameters of the two distributions with respect to two values $\rho_{1}<\rho_{2}$. Here the tests of the four generating hypotheses $\rho \leqq \rho_{i}$, $\rho \geqq \rho_{i}(i=1,2)$ are carried out conditionally, given the value of $X+Y$. The 
conditional distribution of $Y$ given $X+Y=m$ is the binomial distribution $b(p, m)$ corresponding to $m$ trials and probability $p=\mu /(\lambda+\mu)$ of success. The conditional situation is therefore of the kind discussed at the beginning of this section, and leads to the procedure (10.3) with $T=Y$ and $\theta=p$.

Whether $d_{3}$ or $d_{6}$ occurs depends here on $m, d_{3}$ being associated with large values of $m$. To see this, let $F_{p, m}(t)$ denote the conditional cumulative distribution function, given $X+Y=m$, of $Y+U$ where $U$ is independent of $Y$ and is uniformly distributed on $(0,1)$. Then $C_{1}^{\prime}=C^{\prime}(m)$ and $C_{2}=C_{2}(m)$ are determined by

$$
F_{p_{2}, m}\left(C_{2}\right)=1-F_{p_{1}, m}\left(C_{1}^{\prime}\right)=\alpha .
$$

We shall show that $C_{1}^{\prime}\left(m_{1}\right)<C_{2}\left(m_{1}\right)$ implies that also $C_{1}^{\prime}\left(m_{2}\right)<C_{2}\left(m_{2}\right)$ for all $m_{2}>m_{1}$. Since $F_{p_{2}, m}\left(C_{2}\right)=\alpha, F_{p_{1}, m}\left(C_{2}\right)$ is the power of the most powerful level $\alpha$ test for testing $p_{2}$ against $p_{1}$ in a binomial distribution $b(p, m)$. If $C_{1}^{\prime}\left(m_{1}\right)<C_{2}\left(m_{1}\right)$ it follows from (10.9) that in the case of $m$ trials this power is greater than $1-\alpha$; but then it must also exceed $1-\alpha$ for $m_{2}>m_{1}$ trials so that

$$
F_{p_{1}, m_{2}}\left(C_{1}^{\prime}\right)=1-\alpha<F_{p_{1}, m_{2}}\left(C_{2}\right)
$$

and hence $C_{1}^{\prime}\left(m_{2}\right)<C_{2}\left(m_{2}\right)$.

It is interesting to note that in all of these problems the choice between decision $d_{3}$ and $d_{6}$ depends on the distance between $\theta_{1}$ and $\theta_{2}$ relative to the amount of information the data contain for the problem.

While the classification of $\theta$ with respect to a single value $\theta_{0}$, or two values $\theta_{1}<\theta_{2}$, are the most interesting cases, let us consider briefly also the problem of classifying $\theta$ with respect to a countable set of values $\cdots<\theta_{-2}<\theta_{-1}<\theta_{0}<$ $\theta_{1}<\cdots$. This is generated by the problems $P_{ \pm}\left(\theta_{i}\right), i=0, \pm 1, \pm 2, \cdots$. Supposing that $\theta_{i} \rightarrow \pm \infty$ as $i \rightarrow \pm \infty$ and letting $\theta_{-\infty}=-\infty, \theta_{\infty}=\infty$, the possible decisions consist of the totality of statements $\theta_{i}<\theta<\theta_{j}$. The decision $\theta_{i}<\theta<\theta_{j}$ corresponds to the individual decisions that $\theta<\theta_{k}$ for $k \geqq j$ and $\theta>\theta_{k}$ for $k \leqq i$, and that the position of $\theta$ is left in doubt with respect to the points $\theta_{k}$ with $i<k<j$.

The limiting case of this problem, in which one wishes to classify $\theta$ with respect to all possible values of $\theta_{0}$, is obtained by considering simultaneously the problems $\mathcal{P}_{ \pm}\left(\theta_{0}\right)$ for all $\theta_{0}$. The possible decisions then consist of the totality of statements $\underline{\theta}<\theta<\bar{\theta}$, and if $a_{\gamma}=a, b_{\gamma}=b$ for all $\gamma$, (9.3) yields precisely the standard confidence intervals for $\theta$ with 'confidence coefficient $1-2 \alpha$. The loss function resulting from the additivity assumption is in the simplest case ${ }^{2}$

$$
\begin{array}{cl}
(a+b)(\underline{\theta}-\theta)+b(\bar{\theta}-\underline{\theta}) & \text { if } \theta<\underline{\theta} \\
b(\bar{\theta}-\underline{\theta}) & \text { if } \quad \underline{\theta}<\theta<\bar{\theta} \\
(a+b)(\theta-\bar{\theta})+b(\bar{\theta}-\underline{\theta}) & \text { if } \theta>\bar{\theta} .
\end{array}
$$

${ }^{2}$ A loss function of this type was suggested by Wolfowitz [4]. 
More generally one may replace $\bar{\theta}-\underline{\theta}$ by $\int_{\underline{\theta}}^{\theta} d \mu(\theta)$, and similarly for the lengths of the other intervals. Unfortunately the condition of unbiasedness becomes very difficult to interpret in the present problem, and it is doubtful that for the standard distributions the procedure is unbiased with uniformly minimum risk, as is the case with the other problems of this section.

It should be pointed out that all these procedures concerning a single realvalued parameter $\theta$ can be obtained from the standard confidence intervals concerning $\theta$. When classifying $\theta$ with respect to $\theta_{1}$ and $\theta_{2}$ for example, one can state $\theta<\theta_{1}$ if $\bar{\theta}<\theta_{1}$, and $\theta_{1}<\theta<\theta_{2}$ if $\theta_{1}<\underline{\theta}<\bar{\theta}<\theta_{2}$. On the other hand, if $\underline{\theta}<\theta_{1}$ $<\bar{\theta}<\theta_{2}$, only the conclusion $\theta<\theta_{2}$ is possible, the relation of $\theta$ to $\theta_{1}$ being left in doubt. This approach, however, does not yield any optimum properties for these procedures, and does in fact not carry over to problems involving more than one parameter.

In Example (iii) of Sec. 3 we considered the comparison of a number of normal populations $N\left(\theta_{i}, \sigma^{2}\right)$. The consistency difficulties that occurred in combining the decisions $\theta_{i} \leqq \theta_{j}$ for different pairs $(i, j)$ disappear if one treats the problem from the present point of view, which is exactly that from which the problem was treated by Duncan, "Multiple range and multiple $F$-tests," Biometrics, Vol. 11 (1955), pp. 1-42. For each pair $\left(\theta_{i}, \theta_{j}\right)$ the possible decisions are now $\theta_{i}<\theta_{j}$, $\theta_{i}>\theta_{j}$, and $-\infty<\theta_{j}-\theta_{i}<\infty$ instead of the earlier $\theta_{i}=\theta_{j}$. Since there is no loss in omitting the vacuous statements, the total decisions consist in the ordering of some but not necessarily all of the pairs $\left(\theta_{i}, \theta_{j}\right)$. In the case of three populations, for example, the following decision types will occur:
(a) $\theta_{i}<\theta_{j}<\theta_{k}$,
(c) $\theta_{i}<\theta_{j}, \theta_{i}<\theta_{k}$
(b) $\theta_{i}<\theta_{k}, \theta_{j}<\theta_{k}$
(d) $\theta_{i}<\theta_{j}$

(e) no statement.

We shall now show that for this procedure the probability of error can be controlled through the choice of $\alpha$, and that its maximum is in fact attained when all of the $\theta$ 's are equal and is then given by

$$
P\left\{\frac{\left|\bar{X}_{j}-\bar{X}_{i}\right|}{S}>C_{i j} \text { for some } i, j\right\} \text {. }
$$

In the particular case of equal sample sizes this becomes

$$
P\left\{\frac{\max \left|\bar{X}_{j}-\bar{X}_{i}\right|}{S}>C\right\}
$$

where $C$ is the cut-off point of the one-sided $t$-test at level $\alpha{ }^{3}$ The probability of error is the probability measure of the set

$$
\cup\left\{\frac{\bar{X}_{j}-\bar{X}_{i}}{S}>C_{i j}\right\}+\cup\left\{\frac{\bar{X}_{\ell}-\bar{X}_{k}}{S}<C_{k l}\right\}
$$

${ }^{3}$ For a table of the values $C$ for which this probability is 1 per cent or 5 per cent see [2], where also a number of related tables are discussed. 
where the first union is extended over all pairs $(i, j)$ for which $\theta_{j} \leqq \theta_{i}$ and the second union over all pairs $(k, \ell)$ for which $\theta_{\ell} \geqq \theta_{k}$. Let $X_{i}^{*}=X_{i}-\theta_{i}$, so that the $X_{i}^{*}$ are distributed as $N\left(0, \sigma^{2}\right)$. Then $\theta_{j} \geqq \theta_{i}$ and $\bar{X}_{j}-\bar{X}_{i}>C_{i j} S$ imply $X_{j}^{*}-X_{i}^{*}>C_{i j} S$, and $\theta \ell \leqq \theta_{k}$ and $\bar{X}_{\ell}-\bar{X}_{k}<C_{k \ell} S$ imply $X_{\ell}^{*}-X_{k}^{*}<$ $C_{k} \ell S$. The probability of the set (10.9) is therefore not decreased if one replaces the $X^{\prime}$ 's by $X^{*}$ 's, nor if one extends the union over all pairs $(i, j)$ and $(k, \ell)$. But this is equivalent to evaluating the probability of (10.9) under the assumption that all of the $\theta$ 's are equal, which completes the proof.

11. Decision problems with simple loss functions. As a tool for proving the procedures of Secs. 9 and 10 to be unbiased with uniformly minimum risk, we shall now give an extension of Theorem 2 (Sec. 7), which is valid for a rather general class of decision problems. We shall say that a decision problem $\beta$ is simple if it satisfies the following two conditions.

(a) Its loss function $W(\theta, d)$, considered for fixed $d$ as a function of $\theta$, has sets of constancy independent of $d$, that is, there exists a partition $\Pi$ of the parameter space $\Omega$ into sets $\theta_{i}, i \varepsilon I$, such that $W(\theta, d)$ is independent of $\theta$ on each $\theta_{i}$. We may then write

$$
W(\theta, d)=V_{i}(d) \text { for } \theta \varepsilon \theta_{i} .
$$

(b) With respect to some convergence notion in $\Omega, \theta_{n} \rightarrow \theta_{0}$ implies $E_{\theta_{n}} \psi(X) \rightarrow$ $E_{\theta_{0}} \psi(X)$ for each integrable $\psi$ or, if all of the functions $V_{i}$ are bounded, for each bounded $\psi$.

We shall require the following properties of simple decision problems.

(i) For any procedure $\delta$ the risk function $R_{\delta}(\theta)$ is continuous on each set of the partition $\Pi$. The risk function is given by

$$
R_{\delta}(\theta)=E_{\theta} V_{i}[\delta(X)] \text { for } \theta \varepsilon \theta_{i} .
$$

Hence $\theta_{n}, \theta_{0} \varepsilon \theta_{i}$ and $\theta_{n} \rightarrow \theta_{0}$ imply

$$
R_{\delta}\left(\theta_{n}\right)=E_{\theta_{n}} V_{i}[\delta(X)] \rightarrow E_{\theta_{0}} V_{i}[\delta(X)]=R_{\delta}\left(\theta_{0}\right),
$$

as was to be proved.

(ii) Unbiasedness of a procedure $\delta$ implies the continuity of its risk function. By (i) it is enough to prove this for boundary points of $\Pi$. Let $\theta_{0}$ be such a boundary point, and suppose that $\theta_{0} \varepsilon \theta_{i}$ and $\theta_{0}=\lim _{n \rightarrow \infty} \theta_{n}$ with $\theta_{n} \varepsilon \theta_{j}$. Unbiasedness implies

$$
\begin{aligned}
& E_{\theta_{0}} V_{i}[\delta(X)] \leqq E_{\theta_{0}} V_{j}[\delta(X)], \\
& E_{\theta_{n}} V_{i}[\delta(X)] \geqq E_{\theta_{n}} V_{j}[\delta(X)] \quad \text { for } n=1,2, \cdots
\end{aligned}
$$

and hence also

$$
E_{\theta_{0}} V_{i}[\delta(X)] \geqq E_{\theta_{0}} V_{j}[\delta(X)]
$$

It follows that

$$
R_{\delta}\left(\theta_{0}\right)=E_{\theta_{0}} V_{i}[\delta(X)]=E_{\theta_{0}} V_{j}[\delta(X)]=\lim _{n \rightarrow \infty} R_{\delta}\left(\theta_{n}\right) .
$$


(iii) Any restricted product of simple decision procedures is again simple.

We can now prove the main result of this section, which provides a sufficient condition for unbiasedness of a product procedure to imply continuity of the risk functions of all of the component procedures.

(iv) Let $\odot$ be a restricted product of a finite number of simple decision problems $P_{\gamma}$, and suppose that the partitions $\Pi_{\gamma}$ of the component problems inio sets $\theta_{i \gamma}, i \varepsilon I_{\gamma}$ satisfy the following condition.

(*) Let $\theta_{i \gamma_{0}}, \theta_{j \gamma_{0}}$ be any two sets of $\Pi_{\gamma_{0}}$ with common boundary points, let $\theta_{0}$ be any such point, and assume without loss of generality that $\theta_{0} \varepsilon \theta_{i \gamma_{0}}$. Then there exists a sequence of points $\theta_{n} \varepsilon \theta_{j \gamma_{0}}$ such that $\theta_{n} \rightarrow \theta_{0}$ and

$$
\theta_{n} \sim \theta_{0}\left(\Pi_{\gamma}\right) \text { for all } \gamma \neq \gamma_{0},
$$

where $\theta \sim \theta^{\prime}$ (II) indicates that the two points lie in the same set of the partition.

Under these assumptions, if the risk function $R_{\delta}(\theta)$ of a product procedure $\delta$ is continuous, so are the risk functions $R_{\delta_{\gamma}}(\theta)$ of the components $\delta_{\gamma}$ of $\delta$.

To see this, let $\gamma_{0}$ be any fixed value of $\gamma, \theta_{0}$ any boundary point of $\Pi_{\gamma_{0}}$, and $\left\{\theta_{n}\right\}$ the sequence guaranteed by $(*)$. Since $R_{\delta}(\theta)=\sum_{\gamma} R_{\delta_{\gamma}}(\theta)$ is continuous, we have

$$
\sum_{\gamma} R_{\delta_{\gamma}}\left(\theta_{n}\right) \rightarrow \sum_{\gamma} R_{\delta_{\gamma}}\left(\theta_{0}\right)
$$

Also, for each $\gamma \neq \gamma_{0}$ all of the points $\theta_{n}$ lie in the same set of the partition $\Pi_{\gamma}$ with $\theta_{0}$, so that by (i)

$$
R_{\delta_{\gamma}}\left(\theta_{n}\right) \rightarrow R_{\delta_{\gamma}}\left(\theta_{0}\right) \text { for all } \gamma \neq \gamma_{0}
$$

It follows that $R\left(\delta_{\gamma_{0}}, \theta_{n}\right) \rightarrow R\left(\delta_{\gamma_{0}}, \theta_{0}\right)$, as was to be proved, where we have written $R(\delta, \theta)$ for $R_{\delta}(\theta)$.

It is convenient that $(*)$ depends only on the partitions $\Pi_{\gamma}$, not on the values the loss functions $W_{\gamma}$ take on over these partitions. For applications it is further important to note that (*) may be weakened slightly. Let $\Lambda_{i \gamma}$ be the set of common boundary points of $\theta_{i \gamma}$ and $\theta_{j \gamma}$ that belongs to $\theta_{i \gamma}$. Then in order to ensure (iv) it is sufficient if (*) holds on a dense subset of each $\Lambda_{i j \gamma}$. This is an immediate consequence of (i).

Consider now any problem $\rho$, which is a restricted product of a finite number of problems $P_{ \pm}\left(\theta_{\gamma}\right)$, and which satisfies $(*)$. For the component problems continuity of the risk function is equivalent to similarity on the boundary at level $\alpha_{\gamma}=b_{\gamma} /\left(a_{\gamma}+b_{\gamma}\right)$. Hence a product procedure $\delta$ uniformly minimizes the risk among all unbiased procedures of $\odot$ provided each component procedure $\delta_{\gamma}$ uniformly minimizes the risk among all procedures of $\rho_{ \pm}\left(\theta_{\gamma}\right)$ that are similar on the boundary at level $\alpha_{\gamma}$. Since $P_{ \pm}\left(\theta_{\gamma}\right)$ is formally equivalent to the problem of testing $\theta \leqq \theta_{\gamma}$ or $\theta \geqq \theta_{\gamma}$, this is the case in particular if the possible distributions of the observable random variables constitute an exponential family, and the procedures $\delta_{\gamma}$ are the best unbiased tests of the hypotheses in question. Under 
these conditions it is then only necessary to verify $(*)$ in order to establish the desired optimum property for the resulting $\delta$.

As an example consider problem (9.4), which is generated by $\mathcal{P}_{ \pm}\left(\theta_{0}\right)$. Here $P_{-}\left(\theta_{0}\right)$ induces the partition $\theta \leqq \theta_{0}, \theta>\theta_{0}$, with $\theta_{0}$ as its only boundary point. Let $\theta_{n}$ be any sequence of points greater than and tending to $\theta_{0}$. Then all the points $\theta_{n}$ and $\theta_{0}$ lie in the set $\theta \geqq \theta_{0}$, and hence $\theta_{n} \sim \theta_{0}$ with respect to the partition induced by $P_{+}\left(\theta_{0}\right)$, which completes the verification of $(*)$. The argument is exactly the same for problem (9.6) and (10.1).

In the example of Sec. 10 leading to procedure (10.7), a typical partition is $\theta_{2} \leqq \theta_{1}, \theta_{2}>\theta_{1}$. Attention may be restricted to boundary points $\theta^{(0)}$ with coordinates

$$
\theta_{i_{1}}^{0}<\cdots<\theta_{i_{\tau}}^{(0)}<\theta_{1}^{(0)}=\theta_{2}^{(0)}<\theta_{j_{1}}^{(0)}<\cdots<\theta_{j_{s-r-2}}^{(0)} .
$$

For these, $(*)$ is satisfied by the sequence of points $\theta^{(n)}$ with coordinates $\theta_{i}^{(n)}=\theta_{i}^{(0)}$ for $i \neq 2$, and $\theta_{2}^{(n)}$ between $\theta_{1}^{(0)}$ and $\theta_{j_{1}}^{(0)}$ and tending to $\theta_{1}^{(0)}$.

12. Consecutive decisions in a single experiment. The multiple decision problems treated in the previous sections were generated by the simultaneous consideration of a number of simpler component problems. We shall now suppose that these separate problems arise not in parallel but in sequence. A single sample is available for investigating a number of questions that are potentially of interest and are taken up one by one. Whether a given question is relevant, or which of a number of possible alternative formulations is appropriate at a certain stage, depends on the decisions reached up to that point.

(i) As an example suppose that independent variables $X_{1}, \cdots, X_{n}$ from a normal distribution $N\left(\xi, \sigma^{2}\right)$ are measurements on an experimental batch of a new product of quality $\xi$. The product is of no interest unless $\xi>\xi_{0}$, so that one will wish to test first of all the hypothesis $H_{1}: \xi \leqq \xi_{0}$. If the quality is found satisfactory, that is, if $H_{1}$ is rejected, it becomes necessary to investigate the variability of the product. One will then test $H_{2}: \sigma \geqq \sigma_{0}$, and in case this hypothesis is accepted one will try to reduce $\sigma$, for example by using less variable materials. The problem of testing $H_{2}$ arises here only in case $H_{1}$ is rejected.

(ii) Suppose that two treatments are being compared on a number of different categories of patients. Let the observed effect of treatment $i(i=1,2)$ on the $k$ th patient of the $j$ th category be distributed as $N\left(\xi_{i j}, \sigma^{2}\right)$ where

$$
\xi_{i j}=\eta+\lambda_{i}+\mu_{j}+\nu_{i j}\left(\sum_{i} \lambda_{i}=\sum_{j} \beta_{j}=\sum_{i} \nu_{i j}=\sum_{j} \nu_{i j}=0\right) .
$$

Here $\lambda_{i}$ is the main effect of treatment $i$ and $\nu_{i j}$ the interaction between the $i$ th treatment and the $j$ th category. One may believe in the possibility of the interactions being negligible and hence wish first to test the hypothesis $H_{1}: \nu_{i j}=0$ for all $i, j$. In case $H_{1}$ is accepted the $\lambda$ 's are the objects of primary interest, and the problem becomes that of deciding whether $\lambda_{2}-\lambda_{1}$ is $<,=$, or $>0$, or to estimate this difference either by confidence intervals or by a point estimate. On the other hand, if $H_{1}$ is rejected one will be concerned less with the over-all effects of the treatments which is measured by the $\lambda$ 's than with the treatment differences $\xi_{2 j}-\xi_{1 j}$ for each category. 
More generally, let there be given a first problem $\mathcal{P}^{\prime}$, in which the possible decisions are $d_{1}^{\prime}, \cdots, d_{m}^{\prime}$ and the loss function is $W^{\prime}(\theta, d)$. If decision $d_{i}$ is taken, a second problem $\rho_{i}^{\prime \prime}$ arises with possible decisions $d_{i j}^{\prime \prime}\left(j=1, \cdots, n_{i}\right)$ and loss function $W_{i}^{\prime \prime}(\theta, d)$. The combination of these two problems leads to a two-stage problem with decisions $d_{i j}=\left(d_{i}^{\prime}, d_{i j}^{\prime \prime}\right)$. One may of course continue in this manner and suppose that decision $\left(d_{i}^{\prime}, d_{i j}^{\prime \prime}\right)$ gives rise to a further problem $\boldsymbol{P}_{i j}^{\prime \prime \prime}$ with decisions $d_{i j k}^{\prime \prime \prime}$. However, it is enough to treat the case of two levels since the discussion then extends immediately to the more general situation by induction.

In specifying a loss function we shall assume that even if a wrong decision is taken at the first step, so that the second problem is not the most appropriate one or perhaps need not have been considered at all, it is still desirable to do as well with respect to it as is possible. Thus in example (ii) above, if one has incorrectly decided that the interactions are negligible, one will in the estimation of $\theta_{2}-\theta_{1}$ still wish to obtain as good an estimate as possible, and analogously if $H_{1}$ has wrongly been rejected. Whether the assumption holds in Example (i), that is, whether one would wish to control the variability of the new product after having mistakenly judged its quality to be satisfactory, appears to depend on the circumstances of the problem.

With this assumption, a natural loss function for the compound problem is

$$
W\left(\theta, d_{i j}\right)=W^{\prime}\left(\theta, d_{i}^{\prime}\right)+W_{i}^{\prime \prime}\left(\theta, d_{i j}^{\prime \prime}\right) .
$$

The possibility of not considering a second problem in case a certain decision $d_{i}^{\prime}$ is taken at the first step, is included in this formulation. One need then only take as problem $\rho_{i}^{\prime \prime}$ the vacuous decision problem, that is, set $n_{i}=1$ and $W_{i}^{\prime \prime}\left(\theta, d_{i 1}^{\prime \prime}\right)=0$. Suppose in particular, as was the case in Example (i), that a second problem occurs only for one of the decisions of the first stage, say $d_{1}^{\prime}$. The possible decisions of the compound problem are then

and

$$
\begin{aligned}
& d_{i j}=\left(d_{1}^{\prime}, d_{1 j}^{\prime \prime}\right), \\
& d_{2}=d_{2}^{\prime}, \cdots, d_{m}=d_{m}^{\prime},
\end{aligned}
$$$$
j=1, \cdots, n
$$

and the loss function is given by

$$
\begin{array}{ll}
W\left(\theta, d_{i}\right)=W^{\prime}\left(\theta, d_{i}^{\prime}\right), & i=2, \cdots, m ; \\
W\left(\theta, d_{1 j}\right)=W^{\prime}\left(\theta, d_{1}^{\prime}\right)+W^{\prime \prime}\left(\theta, d_{1 j}^{\prime \prime}\right), & j=1, \cdots, n .
\end{array}
$$

Returning now to the general case, suppose that there exist a satisfactory procedure $\delta^{\prime}$ for $\odot^{\prime}$, which takes decision $d_{i}^{\prime}$ when $X \varepsilon D_{i}^{\prime}$, and that the problems $\mathcal{P}_{1}^{\prime \prime}, \cdots, \mathcal{P}_{m}^{\prime \prime}$ are all different. It then seems natural to retain $\delta^{\prime}$ as first step of the compound procedure, and to consider the problems at the second level relative to the circumstances in which they occur, namely conditionally given that $X \in D_{1}^{\prime}, \cdots, X \varepsilon D_{m}^{\prime}$ respectively. Suppose further that for each $i=1$, $\cdots, m$ there exists a satisfactory procedure $\delta_{i}^{\prime \prime}$ for $\mathcal{P}_{i}^{\prime \prime}$ when the distribution of $X$ is the conditional distribution given $X \in D_{i}^{\prime}$. Such a procedure consists of a partition of the new sample space $D_{i}^{\prime}$ into regions $D_{i j}^{\prime \prime}$ in which the decisions 
$d_{i j}^{\prime \prime}$ are taken. Together, the sets $D_{i j}^{\prime \prime}\left(j=1, \cdots, n_{i} ; i=1, \cdots, m\right)$ form a partition of the original sample space and a solution of the compound problem, with decision $d_{i j}=\left(d_{i}^{\prime}, d_{i j}^{\prime \prime}\right)$ being taken when $X \varepsilon D_{i j}^{\prime \prime}$. One can of course again include in the formulation the possibility of ruling out some of the decisions $d_{i j}$, and the resulting compatibility questions can be treated exactly as before. However, this possibility seems to be less important in the present context and in order not to complicate the discussion unnecessarily we shall assume that no restrictions are imposed on the compound decision problem.

As an application consider the case that $m$ and the $n_{i}$ are equal to two, so that each of the component problems is one of hypothesis testing. Suppose that the hypotheses in question concern the parameters $\theta_{i}$ in an exponential family

$$
d P_{\theta}(x)=C\left(\theta_{1}, \cdots, \theta_{r}\right) e^{\Sigma \theta i T_{i}(x)} d \nu(x) .
$$

There then exist uniformly most powerful unbiased tests of the hypotheses $\theta_{i} \leqq \theta_{i}^{0}$ and more generally of $\sum c_{i} \theta_{i} \leqq c_{0}$. (See, for example, [2].) Since after truncation on a fixed set $D$ the family of distributions $P_{\theta}$ retains its property of forming an exponential family, such optimum unbiased tests will in particular also exist at the second stage after a preliminary test of significance has been performed as a first step. This will however not coincide with the standard optimum test for the corresponding problem without truncation.

We have assumed so far that the problems occurring at the second stage are all distinct. Suppose now instead that $\mathcal{P}_{1}^{\prime \prime}$ is appropriate when decisions $d_{1}^{\prime}, \cdots, d_{r_{1}}^{\prime}$ are taken in the first problem, that $\boldsymbol{P}_{2}^{\prime \prime}$ corresponds to decisions $d_{r_{1}+1}^{\prime}, \cdots, d_{r_{1}+r_{2}}^{\prime}$, etc. One would then consider the problems $\mathcal{P}_{1}^{\prime \prime}, \mathcal{P}_{2}^{\prime \prime}, \cdots$ conditionally given that $X \& D_{1}^{\prime}+\cdots+D_{r_{1}}^{\prime}, X \varepsilon D_{r_{1}+1}^{\prime}+\cdots+D_{r_{1}+r_{2}}^{\prime}, \cdots$, and otherwise proceed as before. If one is dealing in particular with the product of two decision problems so that all of the problems at the second level are the same, one considers this common problem $\rho^{\prime \prime}$ given that $X \varepsilon D_{1}^{\prime}+\cdots+D_{m}^{\prime}$, that is, unconditionally. The procedure therefore reduces to the product of the procedures for $\rho^{\prime}$ and $\rho^{\prime \prime}$, so that the present theory agrees with that given earlier for products of decision problems.

Unfortunately the properties of the conditional procedures considered in the present section are not as satisfactory as of those discussed in the earlier parts of this paper. To be specific, let $\odot^{\prime}$ and $\odot_{1}^{\prime \prime}, \cdots, P_{m}^{\prime \prime}$ define a two-stage problem, in which the components $\mathcal{P}_{i}^{\prime \prime}$ of the second stage are distinct. The risk function of a procedure $\delta$ with components $\delta^{\prime}, \delta_{1}^{\prime \prime}, \cdots, \delta_{m}^{\prime \prime}$ is then

$$
R_{\delta}(\theta)=R_{\delta^{\prime}}(\theta)+\sum_{i=1}^{m} P_{\theta}\left(D_{i}^{\prime}\right) R_{\delta_{i}^{\prime \prime} \mid \delta^{\prime}}(\theta)
$$

where the notation $R_{\delta_{i}^{\prime \prime} \mid \delta^{\prime}}$, is used to indicate that this risk component is computed conditionally given $X \in D_{i}^{\prime}$ and hence depends on $\delta^{\prime}$ as well as on $\delta_{i}^{\prime \prime}$.

It is clear from (12.3) that unbiasedness of $\delta^{\prime}$ and $\delta_{1}^{\prime \prime}, \cdots, \delta_{m}^{\prime \prime}$ implies that of $\delta$. Also it is again true for most problems of interest that unbiasedness of $\delta$ implies either unbiasedness or at least similarity on the boundary for $\delta^{\prime}, \delta_{1}^{\prime \prime}$, $\cdots, \delta_{m}^{\prime \prime}$. However, the basic comparison of two procedures in terms of their 
components is no longer simple. In particular,

$$
R_{\delta^{\prime}}(\theta) \leqq R_{\psi^{\prime}}(\theta), \quad R_{\delta_{i}^{\prime \prime} \mid \delta^{\prime}}(\theta) \leqq R_{\psi^{\prime} \mid \psi^{\prime}}(\theta)
$$

does not in general imply $R_{\delta}(\theta) \leqq R_{\psi}(\theta)$. If one wishes to minimize $R_{\delta}(\theta)$ then, given $\delta^{\prime}$, one must select $\delta_{i}^{\prime \prime}$ to minimize $R_{\delta_{i}^{\prime \prime} \mid \delta^{\prime}}(\theta)$. But the best choice of $\delta^{\prime}$ is not necessarily that which minimizes $R_{\delta^{\prime}}(\theta)$ since the choice of $\delta^{\prime}$ influences not only $R_{\delta^{\prime}}(\theta)$ but also the second components of (12.3) and in particular the conditional risks $R_{\delta_{i} \mid \delta^{\prime}}$. As a result of these complications it turns out that for the problem under consideration there usually does not exist among the unbiased procedures one that uniformly minimizes the risk. Of the procedures, the components of which have this optimum property we can only say that they are unbiased, and within the class of all unbiased procedures admissible.

13. Some examples of conditional procedures. Although we have found no satisfactory justification for the procedures discussed in the preceding section, they are rather natural from the Neyman-Pearson point of view, and we shall briefly illustrate them here with a few examples leaving a more detailed discussion and comparison with alternative procedures for a later paper.

(i) The problem mentioned at the beginning of Sec. 12 is concerned with testing, on the basis of a normal sample, the two hypotheses $H_{1}: \xi \leqq \xi_{0}$ and $H_{2}: \sigma \geqq \sigma_{0}$, where $H_{2}$ is assumed to be of interest only in case $H_{1}$ is rejected. If without loss of generality we put $\xi_{0}=0$, the best unbiased procedure for testing $H_{1}$ is Student's $t$-test with rejection region

$$
\bar{X} / S \geqq C
$$

and size $\alpha_{1}=b_{1} /\left(a_{1}+b_{1}\right)$. With this as first step, the condition of unbiasedness implies in the usual way that the rejection region $R_{2}$ of $H_{2}$ must satisfy

$$
P_{\sigma_{0}}\left(R_{2}|S \leqq \bar{x} / C| \bar{x}\right)^{(\bar{x})} \alpha_{2} .
$$

Applying the fundamental lemma of Neyman and Pearson one sees that the uniformly most powerful unbiased conditional test of $\mathrm{H}_{2}$ has a rejection region of the form

$$
S^{2}<f(\bar{X})
$$

Here the function $f$ is defined by

$$
\int_{0}^{f(u)} p_{\sigma_{0}}(z) d z=\alpha_{2} \int_{0}^{u^{2} / C^{2}} p_{\sigma_{0}}(z) d z
$$$$
u>0 \text {, }
$$

where $p_{\sigma_{0}}$ is the probability density of $S^{2}$ when $\sigma=\sigma_{0}$.

The resulting compound procedure then decides between the three possible conclusions

$d_{1}: \xi \leqq \xi_{0}$-the new product is not of satisfactory quality,

$d_{2}: \xi>\xi_{0}, \sigma \geqq \sigma_{0}$-the quality of the new product is satisfactory but its variability must be reduced,

$d_{3}: \xi>\xi_{0}, \sigma<\sigma_{0}$-the new product is satisfactory with regard to both quality and variability, 
as the sample falls into the corresponding one of the regions

$$
\begin{array}{ll}
D_{1}: \bar{X} \mid S \leqq C, & \\
D_{2}: \bar{X} \mid S>C, & S^{2} \geqq f(\bar{X}) \\
D_{3}: \bar{X} \mid S>C, & S^{2}<f(\bar{X}) .
\end{array}
$$

These decision regions are illustrated for the case $n=10, \alpha_{1}=\alpha_{2}=: 05$ in the figure.

(ii) As a somewhat more complex example consider two treatments which are being compared on a number of different categories of patients. Let the observed effect $Y_{i j k}$ of treatment $i(i=1,2)$ on the $k$ th patient $(k=1, \cdots, n)$ in the $j$ th category be distributed as $N\left(\xi_{i j}, \sigma^{2}\right)$, and let

$$
\xi_{i j}=\eta+\lambda_{i}+\mu_{j}+\nu_{i j}\left(\sum_{i} \lambda_{i}=\sum_{j} \mu_{j}=\sum_{i} \nu_{i j}=\sum_{j} \nu_{i j}=0\right)
$$

where $\lambda_{i}$ is the main effect of treatment $i$ and $\nu_{i j}$ the interaction between the $i$ th treatment and $j$ th category. One may here wish to test first the hypothesis of no interaction

$$
H_{1}: \nu_{i j}=0 \quad \text { for all } i, j .
$$

If $H_{1}$ is accepted one will be interested in the difference of the $\lambda$ 's and wish either to test it or alternatively to estimate it by confidence intervals or point estimate. We shall here suppose that we then want to test

$$
H_{2}: \lambda_{2}-\lambda_{1} \leqq 0 .
$$

On the other hand, if $H_{1}$ is rejected one will usually be concerned less with comparing the over-all effects of the two treatments, which is measured by the $\lambda$ 's, than with a comparison of the treatment effects $\xi_{2 j}$ and $\xi_{1 j}$ separately for each category $j$. In particular one may be interested to test the set of hypotheses

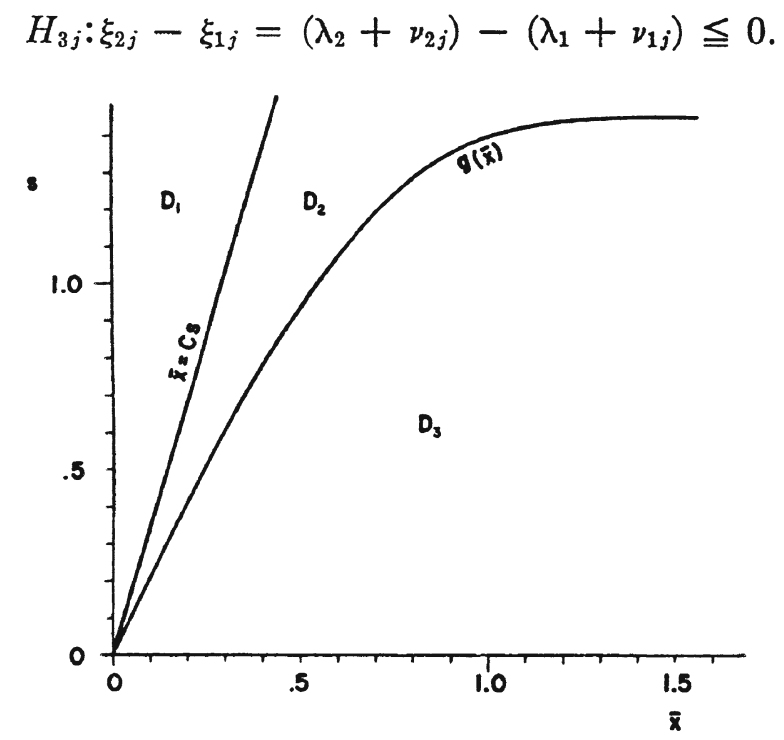

Fig. 1 


\section{E. L. LEHMANN}

Although there exists no uniformly most powerful unbiased test of $H_{1}$ it seems natural to start out with the standard test of this hypothesis which is uniformly most powerful invariant, and is given by the acceptance region

$$
S_{1}^{2} / S_{0}^{2} \leqq C
$$

where

$$
\begin{aligned}
& S_{0}^{2}=\sum \sum \sum\left(Y_{i j k}-Y_{i j .}\right)^{2} \\
& S_{1}^{2}=\sum \sum\left(Y_{i j .}-Y_{i . .}-Y_{. j .}+Y_{\ldots .}\right)^{2} .
\end{aligned}
$$

The hypothesis $H_{2}$ should then be considered conditionally, given $S_{1}^{2} / S_{0}^{2} \leqq C$. A routine application of the theory of unbiased tests and similar regions ${ }^{4}$ shows that a necessary condition for the rejection region $R_{2}$ to be unbiased is

$$
P_{\lambda_{2}=\lambda_{1}}\left\{R_{2} \mid s_{1}^{2}, s_{0}^{2}+s_{2}^{2}, S_{1}^{2} / S_{0}^{2} \leqq C\right\}=\alpha_{2}
$$

for all values of $s_{1}^{2}$ and $s_{0}^{2}+s_{2}^{2}$ where

$$
S_{2}^{2}=\sum_{i=1}^{2}\left(Y_{i . .}-Y_{\ldots . .}\right)^{2}=\frac{1}{2}\left(Y_{2 . .}-Y_{1 . .}\right)^{2} .
$$

If one puts

$$
U=\left(Y_{2 . .}-Y_{1 . .}\right) / S_{0}, \quad V=S_{0}^{2}+S_{2}^{2}, \quad W=\left(S_{0}^{2}+S_{2}^{2}\right) / S_{1}^{2},
$$

it follows from the fundamental lemma that the uniformly most powerful (conditional) test of $\mathrm{H}_{2}$ has the rejection region

$$
U \geqq k(V, W)
$$

where $k$ is determined by

$$
P_{\lambda_{1}=\lambda_{2}}\left\{U \geqq k(V, W) \mid V=v, W=w \text { and } U^{2} \leqq C w-1\right\}^{(v, w)} \alpha_{2} .
$$

Now when $\lambda_{1}=\lambda_{2}$, the variable $U$ is independent of $V$ so that $k$ depends only on $w, k(v, w)=f(w)$ say. The rejection region then becomes

$$
U \geqq f(w)
$$

where $f$ is determined by

$$
P_{\lambda_{1}=\lambda_{2}}\left\{U<f(w) \mid U^{2} \leqq C w-1\right\}^{(w)}\left(1-\alpha_{2}\right) .
$$

Since acceptance of $H_{1}$ is equivalent to $u^{2} \leqq C w-1$ it implies in particular that $0 \leqq C w-1$. The defining condition for $f$ may therefore be written as

$$
\int_{-(C w-1)^{1 / 2}}^{f(w)} p_{U}(u) d u=\left(1-\alpha_{2}\right) \int_{-(C w-1)^{1 / 2}}^{(C w-1)^{1 / 2}} p_{U}(u) d u
$$

where $p_{U}(u)$ is the probability density of $U$ when $\lambda_{1}=\lambda_{2}$, that is, essentially, the density of a $t$-distribution with $2 m(n-1)$ degrees of freedom.

${ }^{4}$ The proof requires the easily shown fact that the family of noncentral $\chi^{2}$-distributions with a fixed number of degrees of freedom is boundedly complete. 
Let us now consider in a similar manner the hypothesis

$$
H_{31}:\left(\lambda_{2}-\lambda_{1}\right)+\left(\nu_{21}-\nu_{11}\right) \leqq 0,
$$

conditionally given that $S_{1}^{2} / S_{0}^{2}>C$. Let $\hat{\lambda}_{i}$ and $\hat{\nu}_{i j}$ be the least squares estimates of the corresponding parameters so that

$$
S_{1}^{2}=\sum_{i=1}^{2} \sum_{j=1}^{m} \hat{\nu}_{i j}^{2}
$$

and let

$$
\begin{aligned}
& Z_{1}=\frac{1}{2}\left(\hat{\lambda}_{2}-\hat{\lambda}_{1}-\hat{\nu}_{21}+\hat{\nu}_{11}\right) \\
& Z_{2}=\frac{1}{2}\left(\hat{\lambda}_{2}-\hat{\lambda}_{1}+\hat{\nu}_{21}-\hat{\nu}_{11}\right) \\
& S_{1}^{\prime 2}=S_{1}^{2}-\left(\hat{\nu}_{21}^{2}-\hat{\nu}_{11}\right)^{2}=S_{1}^{2}-\left(Z_{2}-Z_{1}\right)^{2} .
\end{aligned}
$$

If $\zeta_{i}=E\left(Z_{i}\right)$, the hypothesis becomes $\zeta_{2} \leqq 0$, and unbiasedness of the conditional test of $H_{31}$ with rejection region $R_{31}$ implies

$$
P_{\zeta_{2}=0}\left\{R_{31} \mid z_{1}, s_{0}^{2}+z_{2}^{2}, s_{1}^{\prime 2} \text { and } S_{1}^{2} / S_{0}^{2}>C\right\} \equiv \alpha_{31} .
$$

The condition $S_{1}^{2}>C S_{0}^{2}$ is equivalent to $\left(Z_{2}-Z_{1}\right)^{2}>C S_{0}^{2}-S_{1}^{\prime 2}$, which may be rewritten as

$$
\left(Z_{2}-\frac{Z_{1}}{1+C}\right)^{2}+\frac{C Z_{1}^{2}}{(1+C)^{2}}>\frac{C}{1+C} T^{2}-S_{1}^{\prime 2},
$$

where $T^{2}=S_{0}^{2}+Z_{2}^{2}$. This is satisfied for all values of $Z_{2}$ if

$$
\frac{C}{1+C} T^{2}-S_{1}^{\prime 2}-\frac{C Z_{1}^{2}}{(1+C)^{2}} \leqq 0
$$

and otherwise for the values of $Z_{2}$, for which either

$$
\frac{Z_{2}}{T}>\frac{Z_{1}}{(1+C) T}+\sqrt{\frac{C}{1+C}-\frac{S_{1}^{\prime 2}}{T^{2}}-\frac{C}{(1+C)^{2}} \frac{Z_{1}^{2}}{T^{2}}}=K_{2}\left(\frac{Z_{1}}{T}, \frac{S_{1}^{\prime}}{T}\right)
$$

or

$$
\frac{Z_{2}}{T}<\frac{Z_{1}}{(1+C) T}-\sqrt{\frac{C}{1+C}-\frac{S_{1}^{\prime 2}}{T^{2}}-\frac{C}{(1+C)^{2}} \frac{Z_{1}^{2}}{T^{2}}}=K_{1}\left(\frac{Z_{1}}{T}, \frac{S_{1}^{\prime}}{T}\right) .
$$

Since $Z_{2} / T$ is independent of $Z_{1} / T$ and $S_{1}^{\prime} / T$ when $\zeta_{2}=0$, the uniformly most powerful unbiased test of $H_{31}$ given $S_{1}^{2} / S_{0}^{2}>C$ is then given by the rejection region

$$
\frac{Z_{2}}{T} \geqq K\left(\frac{Z_{1}}{T}, \frac{S_{1}^{\prime}}{T}\right)
$$

with the function $K$ defined as follows. When $\left(Z_{1} / t, S_{1}^{\prime} / t\right)$ satisfies (13.8), we have

$$
K\left(\frac{z_{1}}{t}, \frac{s_{1}^{\prime}}{t}\right)=K
$$


where

$$
\int_{K}^{1} p_{R}(r) d r=\alpha_{31}
$$

$p_{R}(r)$ being the probability density of

$$
R=\frac{Z_{2}}{T}=\frac{\frac{1}{2}\left(\hat{\lambda}_{2}-\hat{\lambda}_{1}-\hat{\nu}_{21}+\hat{\nu}_{11}\right)}{\sqrt{S_{0}^{2}+\frac{1}{4}\left(\hat{\lambda}_{2}-\hat{\lambda}_{1}+\hat{\nu}_{21}-\hat{\nu}_{11}\right)^{2}}}
$$

when $\zeta_{2}=0$. For all other values of $\left(z_{1} / t, s_{1}^{\prime} / t\right), K\left(z_{1} / t, s_{1}^{\prime} / t\right)$ is given by

$$
\begin{aligned}
\int_{K\left(\left(z_{1} / t\right),\left(s_{1}^{\prime} / t\right)\right)}^{1} p_{R}(r) d r=\alpha_{31}\left[\int_{-1}^{\left.K_{1}\left(z_{1} / t\right),\left(s_{1}^{\prime} / t\right)\right)} p_{R}(r) d r\right. & \\
& \left.+\int_{K_{2}\left(\left(z_{2} / t\right),\left(s_{1}^{\prime} / t\right)\right)}^{1} p_{R}(r) d r\right] .
\end{aligned}
$$

(iii) As an example involving more than two stages let us consider the determination of the degree of a regression polynomial. Let $Y_{1}, \cdots, Y_{n}$ be independently normally distributed with constant variance $\sigma^{2}$ and means

$$
\eta_{i}=E\left(Y_{i}\right)=e_{s}+e_{s-1} x_{i}+\cdots+e_{0} x_{i}^{s} \quad(i=1, \cdots, n) .
$$

We shall assume that a polynomial of degree $s$ will in any case be adequate for our purposes, and wish to determine the smallest degree $r \leqq s$ that would also be satisfactory. It is convenient for this purpose to express the regression polynomial in terms of the orthogonal polynomials $P_{i}$ defined by

$$
\sum_{k=1}^{n} P_{i}\left(x_{k}\right) P_{j}\left(x_{k}\right)= \begin{cases}1 & \text { for } i=j \\ 0 & \text { for } i \neq j\end{cases}
$$

Writing

$$
\eta_{i}=c_{s}+c_{s-1} P_{1}\left(x_{i}\right)+\cdots+c_{0} P_{s}\left(x_{i}\right),
$$

we test successively the hypotheses $H_{0}: c_{0}=0, H_{1}: c_{1}=0, \cdots$, continuing as long as no rejection occurs.

The problem can be stated in the following canonical form: $X_{1}, \cdots, X_{s}$ and $S_{0}^{2}$ are independent variables, with $X_{i}$ being distributed as $N\left(\xi_{i}, \sigma^{2}\right)$ and with $S_{0}^{2} / \sigma^{2}$ having a $\chi^{2}$-distribution with $n_{0}=n-s$ degrees of freedom. One wishes to test consecutively the hypotheses $H_{i}: \xi_{i}=0,(i=1, \cdots, s)$ continuing as long as no rejection occurs. Slightly more generally one might have variables $X_{i j}: N\left(\xi_{i j}, \sigma^{2}\right),\left(j=1, \cdots, n_{i} ; i=1, \cdots, s\right)$ and $S_{0}^{2}$ and consider consecutively the hypotheses $H_{i}: \xi_{i j}=0$ for $j=1, \cdots, n_{i}$. Invariance reduces the problem to the statistics $S_{0}^{2}$ and $S_{i}^{2}=\sum_{j=1}^{n_{i}} X_{i j}^{2}$, which are independent and where $S_{i}^{2} / \sigma^{2}$ has a $\chi^{2}$ distribution with $n_{i}$ degrees of freedom when $H_{i}$ is true.

Let

$$
U_{i}=\frac{S_{i}^{2}}{S_{0}^{2}}, \quad V_{i}=\frac{S_{i}^{2}}{S_{i-1}^{2}}, \quad W_{i}=V_{i}\left(1+\frac{1}{U_{i}}\right)=\frac{S_{i}^{2}+S_{0}^{2}}{S_{i-1}^{2}}
$$


We shall now show inductively that the best unbiased conditional test of $H_{i}$, given the acceptance of $H_{1}, \cdots, H_{i-1}$, has an acceptance region of the form

$$
U_{i} \leqq f_{i}\left(W_{i}, V_{i-1}, \cdots, V_{2}\right) \text {. }
$$

The functions $f_{i}$ are defined by

$$
\left\{\begin{array}{l}
f_{1}\left(w_{1}\right)=C, \\
\int_{0}^{f_{i}\left(w_{i}, v_{i-1}, \cdots, v_{2}\right)} p_{i}(r) d r=\left(1-\alpha_{i}\right) \int_{0}^{w_{i} h_{i-1}\left(v_{i-1}, \cdots, v_{2}\right)-1} p_{i}(r) d r \quad(i \geqq 2)
\end{array}\right.
$$

where $p_{i}$ is the probability density of $S_{i}^{2} / S_{0}^{2}$ when $H_{i}$ is true, $h_{i}$ is defined by

$$
\left\{\begin{array}{l}
h_{1}=C, \\
h_{i}\left(v_{i}, \cdots, v_{2}\right)=\min \left[v_{i} h_{i-1}\left(v_{i-1}, \cdots, v_{2}\right), g_{i}\left(v_{i}, \cdots, v_{2}\right)\right] \quad(i \geqq 2)
\end{array}\right.
$$

and $g_{i}$ is a function taking on values in the space of $u_{i}$ and defined by

$$
u=g_{i}\left(v_{i}, \cdots, v_{2}\right) \leftrightarrow v_{i}=\frac{f_{i}^{-1}\left(u, v_{i-1}, \cdots, v_{2}\right)}{1+\frac{1}{u}} \quad(i \geqq 2)
$$

where $f_{i}^{-1}$ denotes the inverse of $f_{i}$ considered for fixed values of $v_{i-1}, \cdots, v_{2}$ as a function of $u$. It is seen that (13.10), (13.11) and (13.12) define $f_{i}$ in terms of $f_{i-1}$.

The best unbiased test of $H_{1}$ has the acceptance region $u_{1} \leqq C$ and hence satisfies (13.9). Suppose now that the acceptance regions $A_{1}, \cdots, A_{i-1}$ have been shown to be given by (13.9). To prove (13.9) for $A_{i}$ we need the fact that

$$
A_{1} \cap \cdots \cap A_{i-1}=\left\{(u, v): u_{i-1} \leqq h_{i-1}\left(v_{i-1}, \cdots, v_{2}\right)\right\}
$$

and since this is true for $i=2$, we may accept (13.13) as part of our induction hypothesis. The condition of unbiasedness implies that $A_{i}$ should satisfy

$P_{B_{i}}\left\{A_{i} \mid s_{0}^{2}+s_{i}^{2}, s_{1}^{2}, \cdots, s_{i-1}^{2}\right.$ and $\left.U_{i-1} \leqq h_{i-1}\left(V_{i-1}, \cdots, V_{2}\right)\right\}=1-\alpha_{i}$. Now $U_{i-1} \leqq h_{i-1}$ is equivalent to

$$
U_{i} \leqq W_{i} h_{i-1}\left(V_{i-1}, \cdots, V_{2}\right)-1
$$

so that the best unbiased acceptance region for $H_{i}$ is

$$
U_{i} \leqq K_{i}\left(W_{i}, S_{1}^{2}, \cdots, S_{i-1}^{2}\right)
$$

where

$$
\begin{aligned}
& P_{B_{i}}\left\{U_{i} \leqq K_{i} \mid s_{0}^{2}+s_{i}^{2}, s_{1}^{2}, \cdots, s_{i-1}^{2} \quad \text { and } \quad U_{i} \leqq W_{i} h_{i-1}\left(V_{i-1}, \cdots, V_{2}\right)-1\right\} \\
& =1-\alpha_{i} .
\end{aligned}
$$

Since $U_{i}$ is independent of $W_{i}, V_{i-1}, \cdots, V_{2}$ when $H_{i}$ is true, it is seen that $K_{i}$ depends only on $w_{i}, v_{i-1}, \cdots, v_{2}$ and that $A_{i}$ is given by (13.9).

To complete the proof, it only remains to verify (13.13) for the set 
$A_{1} \cap \cdots \cap A_{i}$, which is the intersection of

$$
A_{1} \cap \cdots \cap A_{i-1}: u_{i-1} \leqq h_{i-1}\left(v_{i-1}, \cdots, v_{2}\right)
$$

and

$$
A_{i}: u_{\imath} \leqq f_{i}\left(w_{i}, v_{i-1}, \cdots, v_{2}\right)
$$

The inequality describing $A_{i}$ is equivalent to

$$
f_{i}^{-1}\left(u_{i}, v_{i-1}, \cdots, v_{2}\right) \leqq w_{i}=v_{i}\left(1+\frac{1}{u_{i}}\right)
$$

and hence to

$$
A_{i}: u_{i} \leqq g_{i}\left(v_{i}, \cdots, v_{2}\right)
$$

by (13.12). Since the inequality describing $A_{1} \cap \cdots \cap A_{i-1}$ is equivalent to

$$
A_{1} \cap \cdots \cap A_{i-1}: u_{i} \leqq v_{i} h_{i-1}\left(v_{i-1}, \cdots, v_{2}\right),
$$

the intersection $A_{1} \cap \cdots \cap A_{i}$ is given by (13.13), as was to be proved.

A closely related problem arises in the study of components of variance when one is dealing with a hierarchical classification. Here the problem reduces to independent statistics $S_{1}^{2}, S_{2}^{2}, \cdots$ where $S_{i}^{2} / \sigma_{i}^{2}$ has a $\chi^{2}$-distribution with $n_{i}$ degrees of freedom. It follows from the underlying model that $\sigma_{1} \leqq \sigma_{2} \leqq \cdots$, and one is interested in testing successively the hypotheses $H_{1}: \sigma_{2}=\sigma_{1}$, $H_{2}: \sigma_{3}=\sigma_{2}, \cdots$, continuing only if all the previous hypotheses were accepted. If $H_{1}, \cdots, H_{i}$ are true, the distribution of $S_{1}^{2}, \cdots, S_{i}^{2}$ is the same as in the preceding problem, and it is easily seen that exactly the same procedure is applicable to the present situation.

14. Minimizing the maximum risk. For problems of the kind discussed in the preceding section unbiasedness is closely related to the minimax property. We begin by considering the two-decision problem of testing a hypothesis $H: \theta \varepsilon \omega$. Let $d_{0}$ and $d_{1}$ denote the decisions of accepting and rejecting $H$, and let the losses of false rejection and acceptance be $a$ and $b$ respectively. Then we have

(i) A necessary condition for a procedure $\delta$ to be minimax is that it be unbiased.

(ii) This condition is also sufficient if the probability $P_{\theta}(A)$ of any set $A$ is continuous in $\theta$ and if the common boundary of $\omega$ and $\omega^{-1}$ is nonempty.

To see (i) note that unbiasedness of a procedure $\delta_{0}$ implies

$$
P_{\theta}\left(d_{1}\right) \leqq \frac{b}{a+b} \text { for } \theta \varepsilon \omega, \quad P_{\theta}\left(d_{0}\right) \leqq \frac{a}{a+b} \text { for } \theta \varepsilon \omega^{-1} .
$$

Since the risk function of any procedure $\delta$ is given by

$$
R_{\delta}(\theta)= \begin{cases}a P_{\theta}\left(d_{1}\right) & \text { for } \quad \theta \varepsilon \omega \\ b P_{\theta}\left(d_{0}\right) & \text { for } \quad \theta \varepsilon \omega^{-1}\end{cases}
$$


$\delta_{0}$ satisfies

$$
\sup R_{\delta_{0}}(\theta) \leqq \frac{a b}{a+b} .
$$

Any minimax procedure $\delta_{1}$ must then also satisfy (14.3) and hence by (14.2) must also be unbiased. Suppose next that the assumptions of (ii) hold, let $\theta_{0}$ be a common boundary point of $\omega$ and $\omega^{-1}$, and let $\delta_{0}$ be unbiased. Then (14.1) and $P_{\theta}\left(d_{0}\right)=1-P_{\theta}\left(d_{1}\right)$ show that

$$
P_{\theta_{0}}\left(d_{1}\right)=\frac{b}{a+b} \text { and hence } R_{\delta_{0}}\left(\theta_{0}\right)=a b /(a+b) \text {. }
$$

Thus for any unbiased procedure $\delta_{0}$ we have

$$
\sup R_{\delta_{0}}(\theta)=\frac{a b}{a+b} .
$$

By (i), this relation holds in particular for any minimax procedure, so that $a b /(a+b)$ is the minimax risk and (ii) follows from (14.4).

We shall now extend (ii) to the case of a number of hypotheses $H_{i}: \xi_{i}(\theta) \varepsilon \omega_{i}$ $(i=1, \cdots, s)$ to be tested in sequence, where each hypothsis $H_{i}$ is tested only if a particular prescribed chain of decisions has been reached on $H_{1}, \cdots, H_{i-1}$. For these problems we have

THEOREM 3. Every unbiased procedure minimizes the maximum risk provided (i) $P_{\theta}(A)$ is a continuous function of $\theta$ for each $A$, (ii) the $2^{s}$ intersections $\bigcap_{i=1}^{s} \omega_{i}^{x_{i}}$, (each $x_{i}= \pm 1$ ), are all nonempty, (iii) there exists at least one parameter point $\theta_{0}$ which lies on the common boundary of $\omega_{i}$ and $\omega_{i}^{-1}$ for all $i$, (iv) the losses $a_{i}$ and $b_{i}$ for falsely rejecting and accepting $H_{i}$ satisfy condition (14.10) below.

Proof. Let us write $a_{i}^{-1}$ for $b_{i}$, let $d_{i}$ and $d_{i}^{-1}$ denote the decisions of rejecting and accepting $H_{i}$, and let $y_{i}=+1$ if $H_{i+1}$ is considered in case $H_{i}$ is rejected, and $y_{i}=-1$ in the contrary case. If $\theta \varepsilon \bigcap_{i=1}^{s} \omega_{i}^{x_{i}}$, the risk of a procedure $\delta$ is then given by

$$
\begin{aligned}
R_{\delta}(\theta)= & a_{1}^{x_{1}} P\left(d_{1}^{x_{1}}\right)+P\left(d_{1}^{y_{1}}\right)\left\{a_{2}^{x_{2}} P\left(d_{2}^{x_{2}} \mid d_{1}^{y_{1}}\right)+P\left(d_{2}^{y_{2}} \mid d_{1}^{y_{1}}\right)\right. \\
& {\left[a_{3}^{x_{3}} P\left(d_{3}^{x_{3}} \mid d_{1}^{y_{1}} d_{2}^{y_{2}}+\cdots\right]\right\} } \\
= & a_{1}^{x_{1}} P\left(d_{1}^{x_{1}}\right)+a_{2}^{x_{2}} P\left(d_{1}^{y_{1}}\right) P\left(d_{2}^{x_{2}} \mid d_{1}^{y_{1}}\right) \\
& +a_{3}^{x_{3}} P\left(d_{1}^{y_{1}}\right) P\left(d_{2}^{y_{2}} \mid d_{1}^{y_{1}}\right) P\left(d_{3}^{x_{3}} \mid d_{1}^{y_{1}} d_{2}^{y_{2}}\right)+\cdots .
\end{aligned}
$$

By comparing each of these expressions for which $x_{i}=1$ with the corresponding one for which $x_{i}=-1$ but all the other $x$ 's are the same, it is seen that unbiasedness implies and hence is equivalent to the conditions

$$
\begin{aligned}
P_{\theta}\left(d_{i} \mid d_{1}^{y_{1}} \cdots d_{i-1}^{y_{i}-1}\right) & \leqq \frac{b_{i}}{a_{i}+b_{i}} \text { for } \theta \varepsilon \omega_{i} \\
P_{\theta}\left(d_{i}^{1} \mid d_{1}^{y_{1}} \cdots d_{i-1}^{y_{i}-1}\right) & \leqq \frac{a_{i}}{a_{i}+b_{i}} \text { for } \theta \varepsilon \omega_{i}^{-1}
\end{aligned}
$$


for $i=1, \cdots, s$. Putting $r_{i}=a_{i} b_{i} /\left(a_{i}+b_{i}\right)$ we see that the risk function of any unbiased procedure satisfies

$\sup R_{\delta}(\theta) \leqq r_{1}+\frac{a_{1}^{y_{1}}}{a_{1}+b_{1}} r_{2}+\frac{a_{1}^{y_{1}}}{a_{1}+b_{1}} \frac{a_{2}^{y_{2}}}{a_{2}+b_{2}} r_{3}$

$$
+\cdots+\frac{a_{1}^{y_{1}}}{a_{1}+b_{1}} \cdots \frac{a_{s-1}^{y_{s-1}}}{a_{s-1}+b_{s-1}} r_{s}=r^{*} .
$$

It now remains to show that $r^{*}$ is the minimax risk.

Consider any procedure $\delta$, and let

$$
\alpha_{i}=P_{\theta_{0}}\left(d_{i} \mid d_{1}^{y_{1}} \cdots d_{i-1}^{y_{i}-1}\right) .
$$

Then it follows from assumptions (i), (ii) and (iii) that

$$
\sup R_{\delta}(\theta) \geqq \max _{x_{1}, \cdots, x_{s}}\left[\left(a_{1} \alpha_{1}\right)^{x_{1}}+\alpha_{1}^{y_{1}}\left(a_{2} \alpha_{2}\right)^{x_{2}}+\cdots+\alpha_{1}^{y_{1}} \cdots \alpha_{s-1}^{y_{s}-1}\left(a_{s} \alpha_{s}\right)^{x_{x}}\right]
$$

since the $2^{s}$ expressions in brackets are the possible values of $\lim R_{\delta}(\theta)$ as $\theta$ tends to $\theta_{0}$. Let us now minimize the right-hand side of (14.6). Given any $\alpha_{1}, \cdots, \alpha_{s-1}$, $x_{1}, \cdots, x_{s-1}$, the maximum of the pair of expressions

$$
\begin{aligned}
& \left(a_{1} \alpha_{1}\right)^{x_{1}}+\cdots+\alpha_{1}^{y_{1}} \cdots \alpha_{s-2}^{y_{s}-2}\left(a_{s-1} \alpha_{s-1}\right)^{x_{s-1}}+\alpha_{1}^{y_{1}} \cdots \alpha_{s-1}^{y_{s-1}}\left(a_{s} \alpha_{s}\right), \\
& \left(a_{1} \alpha_{1}\right)^{x_{1}}+\cdots+\alpha_{1}^{y_{1}} \cdots \alpha_{s-2}^{y_{s-2}}\left(a_{s-1} \alpha_{s-1}\right)^{x_{s-1}}+\alpha_{1}^{y_{1}} \cdots \alpha_{s-1}^{y_{s-1}}\left(a_{s} \alpha_{s}\right)^{-1},
\end{aligned}
$$

is clearly minimized by minimizing

$$
\max \left[a_{s} \alpha_{s}, a_{s}^{-1}\left(1-\alpha_{s}\right)\right] .
$$

This gives $\alpha_{s}=\alpha_{s}^{*}$ where we put

$$
\alpha_{i}^{*}=\frac{a_{i}^{-1}}{a_{i}+\overline{a_{i}^{-1}}}=\frac{b_{i}}{a_{i}+b_{i}}
$$

so that

$$
a_{i} \alpha_{i}^{*}=\left(a_{i} \alpha_{i}^{*}\right)^{-1} .
$$

We can now proceed inductively. Suppose that it has already been shown for any fixed $\alpha_{1}, \cdots, \alpha_{i}$ that the right-hand side of (14.6) is minimized by $\alpha_{j}=\alpha_{j}^{*}$ for $j=i+1, \cdots, s$. Consider now the minimization of the maximum of the quantities

$$
\begin{gathered}
\left(a_{1} \alpha_{1}\right)^{x_{1}}+\cdots+\alpha_{1}^{y_{1}} \cdots \alpha_{i-1}^{y_{i}-1}\left(\alpha_{2} \alpha_{i}\right)+\alpha_{1}^{y_{1}} \cdots \alpha_{i}^{y_{i}}\left[\left(a_{i+1} \alpha_{i+1}^{*}\right)^{x_{i+1}}\right. \\
\left.+\left(\alpha_{i+1}^{*}\right)^{y_{i+1}}\left(a_{i+1} \alpha_{i+2}^{*}\right)^{x_{i+2}}+\cdots\right] \\
\left(a \alpha_{1}\right)^{x_{1}}+\cdots+a_{1}^{y_{1}} \cdots \alpha_{i-1}^{y_{i}-1}\left(a_{i} \alpha_{i}\right)^{-1}+\alpha_{1}^{y_{1}} \cdots \alpha_{i}^{y_{i}} \\
{\left[\left(a_{i+1} \alpha_{i+1}^{*}\right)^{x_{i+1}}+\left(\alpha_{i+1}^{*}\right)^{y_{i+1}}\left(a_{i+2} \alpha_{i+2}^{*}\right)^{y_{i+2}}+\cdots\right]}
\end{gathered}
$$

where the expression in brackets by (14.8) is independent of the $x$ 's and hence equal to 


$$
k_{i}=a_{i+1} \alpha_{i+1}^{*}+\left(\alpha_{i+1}^{*}\right)^{y_{i+1}} a_{i+2} \alpha_{i+2}^{*}+\cdots+\left(\alpha_{i+1}^{*}\right)^{y_{i+1}} \cdots\left(\alpha_{s-1}^{*}\right)^{y_{i-1}} a_{s} \alpha_{s}^{*} .
$$

Eliminating the common terms at the beginning, and the common factor $\alpha_{1}^{y_{1}} \cdots \alpha_{i-1}^{y_{i}-1}$ of the terms involving $\alpha_{i}$, we see that it is enough to minimize

$$
\max \left[a_{i} \alpha_{i}+k_{i} \alpha_{i}^{y_{i}}, b_{i} \alpha_{i}^{-1}+k_{i} \alpha_{i}^{y_{i}}\right] .
$$

This is achieved by equating the two quantities, which gives $\alpha_{i}=\alpha_{i}^{*}$, provided the coefficient of $\alpha_{i}$ in $b_{i}+\left(k_{i}-b_{i}\right) \alpha_{i}$ is $<0$ in case $y_{i}=1$, or that the coefficient of $\alpha_{i}$ in $a_{i} \alpha_{i}+k_{i}\left(1-\alpha_{i}\right)$ is $>0$ in case $y_{i}=-1$. Thus the right-hand side of (14.6) is minimized by putting $\alpha_{i}=\alpha_{i}^{*}$ for all $i$, provided

$$
\begin{array}{r}
a_{i+1} \alpha_{i+1}^{*}+\left(\alpha_{i+1}^{*}\right)^{y_{i+1}} a_{i+2} \alpha_{i+2}^{*}+\cdots+\left(\alpha_{i+1}^{*}\right)^{y_{i+1}} \cdots\left(\alpha_{s-1}^{*}\right)^{y_{s-1}} a_{s} \alpha_{s}^{*} \\
<a_{i}^{-y_{i}} .
\end{array}
$$

Putting $\alpha_{i}=\alpha_{i}^{*}$ in the right-hand side of (14.6) one then sees that the $2^{8}$ quantities in brackets become equal, and that their common value is $r^{*}$. Thus, for any procedure $\delta$, we have

$$
\sup R_{\delta}(\theta) \geqq r^{*}
$$

and the desired result now follows from comparison with (14.5).

Corollary. The conclusion of Theorem 3 holds if (14.10) is replaced by either

$$
y_{i}=1 \text { for all } i \text { and } b_{1} \geqq b_{2} \geqq \cdots \geqslant b_{s}
$$

or

$$
y_{i}=-1 \text { for all } i \text { and } a_{1} \geqq a_{2} \geqq \cdots \geqq a_{s} \text {. }
$$

Proof. It is necessary only to show that each of the conditions (14.11) and (14.12) implies (14.10). If all the $y$ 's are +1 the left-hand side of (14.10) may be rewritten as

$$
b_{i+1}\left(\alpha_{i+1}^{*}\right)^{-1}+b_{i+2}\left(\alpha_{i+2}^{*}\right)^{-1} \alpha_{i+1}^{*}+\cdots+b_{s}\left(\alpha_{s}^{*}\right)^{-1} \alpha_{i+1}^{*} \cdots \alpha_{s-1}^{*} .
$$

If (14.11) holds, this is

$$
\begin{aligned}
& \leqq b_{i+1}\left[\left(\alpha_{i+1}^{*}\right)^{-1}+\alpha_{i+1}^{*}\left(\alpha_{i+2}^{*}\right)^{-1}+\cdots+\alpha_{i+1}^{*} \cdots \alpha_{s-1}^{*}\left(\alpha_{s}^{*}\right)^{-1}\right] \\
& =b_{i+1}\left[1-\alpha_{i+1}^{*} \alpha_{i+2}^{*} \cdots \alpha_{s}^{*}\right]<b_{i+1} \leqq b_{i} .
\end{aligned}
$$

Similarly, if (14.12) holds, the left-hand side of (14.10) is

$$
\begin{aligned}
& \leqq a_{i+1}\left[\alpha_{i+1}^{*}+\left(\alpha_{i+1}^{*}\right)^{-1} \alpha_{i+2}^{*}+\cdots+\left(\alpha_{i+1}^{*} \cdots \alpha_{s-1}^{*}\right)^{-1} \alpha_{s}^{*}\right] \\
& =a_{i+1}\left[1-\left(\alpha_{i+1}^{*} \cdots \alpha_{s}^{*}\right)^{-1}\right]<a_{i} .
\end{aligned}
$$

If the assumptions of Theorem 3 are not satisfied it is sometimes possible to prove a slightly weaker result. We shall illustrate this with the simplest case of Example (ii) of Sec. 12. Here one is concerned with four means $\xi_{i j}(i, j=1,2)$ given by

$$
\begin{array}{ll}
\xi_{11}=\lambda+\mu+\nu, & \xi_{12}=\lambda-\mu-\nu \\
\xi_{21}=-\lambda+\mu-\nu, & \xi_{22}=-\lambda-\mu-\nu .
\end{array}
$$


The first hypothesis tested is $H_{0}: \nu=0$. In case of acceptance this is followed by $H_{1}: \lambda \leqq 0$, while in case of rejection one is interested in the two hypotheses $H_{2}: \xi_{11} \leqq \xi_{21}$ which is equivalent to $\lambda+\nu \leqq 0$ or $H_{3}: \xi_{12} \leqq \xi_{22}$ which is equivalent to $\lambda-\nu \leqq 0$. The feature which complicates this problem is that when $H_{0}$ is true the three remaining hypotheses must either all be true or all be false. Thus the condition corresponding to (ii) of Theorem 3 is not satisfied.

Let us denote the decision of rejecting or accepting $H_{i}$ by $d_{i}$ and $d_{i}^{-1}$ as before, and consider the class $\mathfrak{S}_{0}$ of procedures satisfying

$$
\begin{aligned}
& P\left(d_{0}\right) \leqq \frac{b_{0}}{a_{0}+b_{0}} \quad \text { if } \quad \nu=0, \quad P\left(d_{0}^{-1}\right) \leqq \frac{a_{0}}{a_{0}+b_{0}} \quad \text { if } \quad \nu \neq 0 \\
& P\left(d_{1} \mid d_{0}^{-1}\right) \leqq \frac{b_{1}}{a_{1}+b_{1}} \quad \text { if } \quad \lambda+\nu \leqq 0 \text {, } \\
& P\left(d_{1}^{-1} \mid d_{0}^{1}\right) \geqq \frac{a_{1}}{a_{1}+b_{1}} \text { if } \lambda+\nu>0 \\
& P\left(d_{i} \mid d_{0}\right) \leqq \frac{b_{i}}{a_{i}+b_{i}} \text { if } H_{i} \text { is true, } \\
& P\left(d_{i}^{-1} \mid d_{0}\right) \leqq \frac{a_{i}}{a_{i}+b_{i}} \text { if } H_{i} \text { is false } \quad(i=2,3),
\end{aligned}
$$

We shall then prove that any element of $\mathfrak{S}_{0}$, which is a subclass of the class of unbiased procedures, is minimax, under the additional assumption that

$$
a_{i}=a \text { and } b_{i}=b \text { for all } i \text {. }
$$

For any procedure $\delta$, consider the error probabilities $\alpha_{0}=P\left(d_{0}\right), \alpha_{1}=P\left(d_{1} \mid d_{0}{ }^{1}\right)$ $\alpha_{i}=P\left(d_{i} \mid d_{0}\right)(i=2,3)$, evaluated for $\lambda=\nu=0$ and some fixed values $\mu_{0}$ and $\sigma_{0}$ of $\mu$ and $\sigma$. Then the possible value of $\lim R_{\delta}(\theta)$ as $\theta=(\lambda, \mu, \nu, \sigma)$ tends to $\theta_{0}=\left(0, \mu_{0}, 0, \sigma_{0}\right)$ are

$$
\begin{array}{ll}
a_{1}\left(1-\alpha_{0}\right) \alpha_{1}+\alpha_{0}\left[a_{0}+a_{2} \alpha_{2}+a_{3} \alpha_{3}\right] & \text { for } \theta \varepsilon \omega_{0} \omega_{1} \omega_{2} \omega_{3} \\
b_{1}\left(1-\alpha_{0}\right)\left(1-\alpha_{1}\right)+\alpha_{0}\left[a_{0}+b_{2}\left(1-\alpha_{2}\right)+b_{3}\left(1-\alpha_{3}\right)\right]
\end{array}
$$

for $\theta \varepsilon \omega_{0} \omega_{1}^{-1} \omega_{2} \omega_{3}^{-1}$

$$
\begin{aligned}
& \left(1-\alpha_{0}\right)\left[b_{0}+a_{1} \alpha_{1}\right]+\alpha_{0}\left[a_{2} \alpha_{2}+a_{3} \alpha_{3}\right] \\
& \left(1-\alpha_{0}\right)\left[b_{0}+a_{1} \alpha_{1}\right]+\alpha_{0}\left[a_{2} \alpha_{2}+b_{3}\left(1-\alpha_{3}\right)\right] \\
& \left(1-\alpha_{0}\right)\left[b_{0}+a_{1} \alpha_{1}\right]+\alpha_{0}\left[b_{2}\left(1-\alpha_{2}\right)+a_{3} \alpha_{3}\right] \\
& \left(1-\alpha_{0}\right)\left[b_{0}+a_{1} \alpha_{1}\right]+\alpha_{0}\left[b_{2}\left(1-\alpha_{2}\right)+b_{3}\left(1-\alpha_{3}\right)\right]
\end{aligned}
$$
for $\theta \varepsilon \omega_{0}^{-1} \omega_{1} \omega_{2} \omega_{3}$ for $\theta \varepsilon \omega_{0}^{-1} \omega_{1} \omega_{2} \omega_{3}^{-1}$ for $\theta \varepsilon \omega_{0}^{-1} \omega_{1} \omega_{2}^{-1} \omega_{3}$

for $\theta \varepsilon \omega_{0}^{-1} \omega_{1} \omega_{2}^{-1} \omega_{3}^{-1}$

$$
\left(1-\alpha_{0}\right)\left[b_{0}+b_{1}\left(1-\alpha_{1}\right)\right]+\alpha_{0}\left[a_{2} \alpha_{2}+a_{3} \alpha_{3}\right]
$$

for $\theta \varepsilon \omega_{0}^{-1} \omega_{1}^{-1} \omega_{2} \omega_{3}$ 


$$
\begin{array}{cc}
\left(1-\alpha_{0}\right)\left[b_{0}+b_{1}\left(1-\alpha_{1}\right)\right]+\alpha_{0}\left[a_{2} \alpha_{2}+b_{3}\left(1-\alpha_{3}\right)\right] & \text { for } \theta \varepsilon \omega_{0}^{-1} \omega_{1}^{-1} \omega_{2} \omega_{3}^{-1} \\
\left(1-\alpha_{0}\right)\left[b_{0}+b_{1}\left(1-\alpha_{1}\right)\right]+\alpha_{0}\left[b_{2}\left(1-\alpha_{2}\right)+a_{3} \alpha_{3}\right] & \text { for } \theta \varepsilon \omega_{0}^{-1} \omega_{1}^{-1} \omega_{2}^{-1} \omega_{3} \\
\left(1-\alpha_{0}\right)\left[b_{0}+b_{1}\left(1-\alpha_{1}\right)\right]+\alpha_{0}\left[b_{2}\left(1-\alpha_{2}\right)+b_{3}\left(1-\alpha_{3}\right)\right] & \text { for } \theta \varepsilon \omega_{0}^{-1} \omega_{1}^{-1} \omega_{2}^{-1} \omega_{3}^{-1} .
\end{array}
$$

With $u=\alpha_{0}, v=\left(1-\alpha_{0}\right) \alpha_{1}, w=\alpha_{0} \alpha_{2}, z=\alpha_{0} \alpha_{3}$ and $a_{i}=a, b_{i}=b$ these quantities become

$$
\begin{array}{r}
a[u+v+w+z] \\
(a+b) u-b v-b w-b z+b \\
-b u+a v+a w+a z+b \\
a v+a w-b z+b \\
a v-b w+a z+b \\
b u+a v-b w-b z+b \\
-2 b u-b v+a w+a z+2 b \\
-b u-b v+a w-b z+2 b \\
-b u-b v-b w+a z+2 b \\
-2 b u-b v-b w-b z+2 b .
\end{array}
$$

From the first form of these 10 quantities it is seen that they all become equal for $\alpha_{i}=\alpha_{i}^{*}=b_{i} /\left(a_{i}+b_{i}\right)$. Let the corresponding values of $(u, v, w, z)$ be $\left(u_{0}\right.$, $\left.v_{0}, w_{0}, z_{0}\right)$. We shall now show that if we change from $\left(u_{0}, v_{0}, w_{0}, z_{0}\right)$ to some other point $\left(u_{1}, v_{1}, w_{1}, z_{1}\right)$ at least one of the 10 quantities will be increased so that $\left(u_{0}, v_{0}, w_{0}, z_{0}\right)$ and hence $\alpha_{i}=\alpha_{i}^{*}$ minimizes their maximum. If in (14.15) all possible sign combinations were occurring, this would be obvious. For then in at least one row all of the four increments $\pm\left(u_{1}-u_{0}\right), \pm\left(v_{1}-v_{0}\right), \pm\left(w_{1}-w_{0}\right)$, $\pm\left(z_{1}-z_{0}\right)$ would be positive. But of the 16 possible combinations only 12 occur (with rows 4 and 5 each counting for two combinations, the missing combinations being,,,-+--+-+++-+-+-+

As an example let us consider the first possibility. Suppose that $u_{1}=u_{0}-\xi$, $v_{1}=v_{0}+\eta, w_{1}=w_{0}-\Delta, z_{1}=z_{0}-\zeta$, with $\xi, \eta, \Delta, \zeta$ all nonnegative. The total change in the first and tenth rows of (14.15) will be

$$
a(-\xi+\eta-\Delta-\zeta) \text { and } b(2 \xi-\eta+\Delta+\zeta) .
$$

Since both of these are to be $\leqq 0$, we have

$$
2 \xi+\Delta+\zeta \leqq \eta \leqq \xi+\Delta+\zeta
$$


and hence $\xi=0$. But then the change in the sixth row will be positive unless also $\eta=\Delta=\zeta=0$. The other three possibilities can be ruled out in a similar manner so that $\alpha_{i}=\alpha_{i}^{*}$ minimizes the maximum of the 10 quantities in question. If $r^{*}$ denotes the common value $\alpha_{0}^{*}\left[a_{0}+a_{1} \alpha_{1}^{*}+a_{2} \alpha_{2}^{*}+a_{3} \alpha_{3}^{*}\right]$ of these quantities, it only remains to show that (14.13) implies $R_{\delta}(\theta) \leqq r^{*}$ for all $\theta$. This is clearly the case since for each $i$ the coefficient of $\alpha_{i}$ in (14.14) is $\geqq 0$ in the lines corresponding to $\theta \varepsilon \omega_{i}$ and $\leqq 0$ otherwise.

The result that the natural combination of a number of best unbiased or minimax tests leads to a minimax procedure for the compound problem, does unfortunately not hold even in the simplest cases in which the different hypotheses concern the same parameter. Consider for example problem (i) of section 3, in which the simultaneous consideration of $H_{1}: \theta \geqq \theta_{0}$ and $H_{2}: \theta \leqq \theta_{0}$ leads to the choice between the three decisions $d_{2}: \theta<\theta_{0}, d_{0}: \theta=\theta_{0}$ and $d_{1}: \theta>\theta_{0}$. If the losses of false rejection and acceptance are $a$ and $b$ for both hypotheses the risk function is given by

$$
R_{\delta}(\theta)= \begin{cases}b P_{\theta}\left(d_{0}\right)+(a+b) P_{\theta}\left(d_{1}\right) & \text { for } \theta<\theta_{0} \\ a\left[P_{\theta}\left(d_{1}\right)+P_{\theta}\left(d_{2}\right)\right] & \text { for } \theta=\theta_{0} \\ b P_{\theta}\left(d_{0}\right)+(a+b) P_{\theta}\left(d_{2}\right) & \text { for } \theta>\theta_{0} .\end{cases}
$$

If $\alpha_{i}=P_{\theta_{0}}\left(d_{i}\right)$ we have

(14.16) $\quad \sup R_{\delta}(\theta) \geqq \max \left[a \alpha_{1}-b \alpha_{2}+b, a\left(\alpha_{1}+\alpha_{2}\right), a \alpha_{2}-b \alpha_{1}+b\right]$.

For a given value of $\alpha_{1}+\alpha_{2}$, the maximum of the first and third terms on the right-hand side is minimized by equating them which gives $\alpha_{1}=\alpha_{2}$ and reduces the right-hand side of (14.16) to

$$
\max [(a-b) \alpha+b, 2 a \alpha] \text {. }
$$

In the usual case that $a>b$, this is minimized for $\alpha=0$, that is, for $\alpha_{0}=1$, $\alpha_{1}=\alpha_{2}=0$. In the standard case that the family $P_{\theta}$ is homogeneous this implies $P_{\theta}\left(d_{0}\right)=1$ for all $\theta$, the risk function of which is

$$
R_{\delta}(\theta)=\left\{\begin{array}{lll}
b & \text { if } & \theta \neq \theta_{0} \\
0 & \text { if } & \theta=\theta_{0}
\end{array}\right.
$$

which is then clearly minimax.

\section{REFERENCES}

[1] E. L. Lehmann, "A theory of some multiple decision problems. I.," Am. Math. Stat., Vol. 28 (1957), pp. 1-25.

[2] E. L. Lehmann and H. Schefft, "Completeness, similar regions and unbiased estimation," Sankhyā, Vol. 10 (1950), pp. 305-339.

[3] Joтce M. MAx, "Extended and corrected tables of the upper percentage points of the Studentized range," Biometrika, Vol. 39 (1952), pp. 192-193.

[4] J. Wolfowitz, "Minimax estimates of the mean of a normal distribution with known variance," Ann. Math. Stat., Vol. 21 (1950), pp. 218-230. 